\title{
Direct numerical evaluation of multi-loop integrals without contour deformation
}

\author{
Roberto Pittau ${ }^{1,2, a}$, Bryan Webber ${ }^{3, b}$ \\ ${ }^{1}$ Departamento de Física Teórica y del Cosmos and CAFPE, Universidad de Granada, 18071 Granada, Spain \\ 2 Theoretical Physics Department, CERN, 1211 Geneva 23, Switzerland \\ ${ }^{3}$ University of Cambridge, Cavendish Laboratory, J.J. Thomson Avenue, Cambridge, UK
}

Received: 1 November 2021 / Accepted: 6 January 2022 / Published online: 20 January 2022

(C) The Author(s) 2022

\begin{abstract}
We propose a method for computing numerically integrals defined via $i \epsilon$ deformations acting on single-pole singularities. We achieve this without an explicit analytic contour deformation. Our solution is then used to produce precise Monte Carlo estimates of multi-scale multi-loop integrals directly in Minkowski space. We corroborate the validity of our strategy by presenting several examples ranging from one to three loops. When used in connection with fourdimensional regularization techniques, our treatment can be extended to ultraviolet and infrared divergent integrals.
\end{abstract}

\section{Introduction}

The ever-increasing precision of data from particle physics experiments requires a comparable or better level of precision in theoretical predictions, both to establish the parameters of the Standard Model and to search for physics beyond it. To achieve such precision requires the computation of multiloop amplitudes. A fundamental ingredient of such calculations is the evaluation of master loop integrals (MIs), in terms of which the problem is reduced. This can be performed by analytic, semi-numerical or fully numerical techniques (see [1] for a recent review). Analytic methods are very successful when the class of functions that contribute to the result is known, which usually happens when the number of internal and external masses is limited. However, such a-priori knowledge is not always available, especially when the number of scales increases, so that in these cases one would like to be able to compute MIs numerically, for instance by Monte Carlo (MC) techniques.

In the numerical computation of MIs, an important problem is the appearance of integrable threshold singularities, where single poles are moved away from the real integration domain by the $i \epsilon$ prescription. These singularities require special treatment, such as a contour deformation into the complex plane [2-5], or vanishing-width extrapolations methods [6-9]. Contour deformations are usually controlled by some parameter whose value should be not too small, to guarantee numerical accuracy, and not too large, to avoid crossing branch cuts. In extrapolation methods a series of integrals should be determined that converges to the right value while keeping the computation time low.

This paper explains how integrals defined through the $i \epsilon$ prescription acting on first-order poles can be evaluated numerically without deforming the integration contour into the complex plane, and how this can be employed to compute MIs appearing in multi-loop calculations. In addition, we demonstrate that this strategy allows one to compute recursively higher-loop functions in terms of lower-loop ones. Other semi-numerical methods relying on one-looplike objects to build higher loops can be found in [10-12]. In [10] a Wick rotation of the loop momentum is needed to avoid singularities. The Feynman parameter space is used in [11], and a contour deformation in [12]. Our method works directly in Minkowski space and avoids contour deformations.

The structure of the paper is as follow. Section 2 details our approach. In Sect. 3 we use it to integrate numerically threshold singularities after an analytic integration over the energycomponents of the loop momenta. Section 4 explains how to glue together lower-loop structures to compute numerically certain classes of higher-loop MIs. Finally, in Sects. 5 and 6 we extend our treatment to ultraviolet and infrared divergent configurations regularized via the four-dimensional method of [13].

\footnotetext{
a e-mail: pittau@ugr.es (corresponding author)

be-mail: webber@hep.phy.cam.ac.uk
} 


\section{Avoiding contour deformation}

In this section we present two methods which avoid contour deformation. The first method uses complex analysis, while the second approach directly works with the original integrand. The two procedures are equivalent, in that they give rise to the same mappings. The numerical results presented in the paper are obtained with method 1 and cross-checked with method 2 .

\subsection{Method 1}

For the sake of clarity, distinct letters (with or without additional subscripts) are used to denote variables ranging in different intervals. In particular, we employ $x$ when $-1 \leq x \leq$ $1, y$ if $0 \leq y \leq 1, \sigma$ provided $-\infty<\sigma<\infty$. Finally, $0 \leq \rho \leq 1$ stands for a random Monte Carlo (MC) variable.

The core of the procedure is a change of variable such that the $1 /(x+i \epsilon)$ behaviour of the integral

$I:=\lim _{\epsilon \rightarrow 0} \int_{-1}^{1} d x \frac{1}{x+i \epsilon}$

is flattened with $x \in \mathbb{R}$. This is obtained by imposing

$x+i \epsilon=e^{i \pi(1-z)}$

where $z$ is a new complex integration variable. In fact, inserting (2) in (1) gives the desired result,

$I=-i \pi f_{0}^{1} d z$

Equation (3) evaluates to $-i \pi$ along any curve in the $z$ complex plane connecting $z=0$ to $z=1$ when $\epsilon \rightarrow 0$. We use this freedom to impose $x \in \mathbb{R}$ by parametrizing

$z=\alpha+i \beta$ with $\alpha, \beta \in \mathbb{R}$ and $\frac{\epsilon}{\pi} \leq \alpha \leq 1-\frac{\epsilon}{\pi}$.

Inserting (4) in (2) gives

$x=e^{\pi \beta} \cos [\pi(1-\alpha)]+i\left\{e^{\pi \beta} \sin [\pi(1-\alpha)]-\epsilon\right\}$,

which is real when $\pi \beta=\ln \frac{\epsilon}{\sin [\pi(1-\alpha)]}$, namely

$x=x_{\alpha}:=\frac{\epsilon}{\tan [\pi(1-\alpha)]}$.

Therefore

$d z=d \alpha\left(1+i \frac{d \beta}{d \alpha}\right)$

which gives

$f_{0}^{1} d z=\lim _{\epsilon \rightarrow 0} \frac{1}{g_{\epsilon}} \int_{\epsilon / \pi}^{1-\epsilon / \pi} d \alpha\left(1+i \frac{x_{\alpha}}{\epsilon}\right), g_{\epsilon}:=1-\frac{2 \epsilon}{\pi}$, where $g_{\epsilon}$ has been introduced to impose the normalization to 1 also for small but not vanishing values of $\epsilon$. In summary, after changing variable as in (2), the requirement $x \in \mathbb{R}$ determines the relation between $\Re e(z)$ and $\Im m(z)$.

Armed with these results, we generalize (1) to an integration over a function

$f(x)=\phi(x) /(x+i \epsilon)$,

with $\phi(x)$ sufficiently smooth at $x=0,{ }^{1}$

$I_{f}:=\int_{-1}^{1} d x f(x)=\int_{0}^{1} d y[f(-y)+f(y)]$.

Splitting the integration region of (8) into the two sectors with $x_{\alpha}<0$ or $x_{\alpha}>0$ gives

$$
\begin{aligned}
I_{f}= & -\frac{i \pi}{g_{\epsilon}} \int_{\epsilon / \pi}^{1 / 2} d \alpha \\
& \times\left[\left(1-i \frac{y_{\alpha}}{\epsilon}\right) \phi\left(-y_{\alpha}\right)+\left(1+i \frac{y_{\alpha}}{\epsilon}\right) \phi\left(y_{\alpha}\right)\right],
\end{aligned}
$$

where $y_{\alpha}:=\epsilon / \tan (\alpha \pi)$. Equation (11) can be translated to a MC language by looking for the local density $g(y)$ that corresponds to a change of variable $d \rho=g(y) d y$ reabsorbing the singular behaviour of the integrand of (10),

$I_{f}=\int_{0}^{1} d \rho \frac{f(-y)+f(y)}{g(y)}$,

with $\int_{0}^{1} d y g(y)=1$. By comparing (12) to (11) one determines

$g(y)=\frac{2 \epsilon}{\pi\left(y^{2}+\epsilon^{2}\right)}, \quad y=\frac{\epsilon}{\tan (\alpha \pi)}, \quad \alpha=\frac{\epsilon}{\pi}+\frac{\rho g_{\epsilon}}{2}$.

The mapping of (13) optimizes the integration over the real part of $z$ (see (7)). This gives stable numerical results when $\phi(x)$ is such that the $y_{\alpha} / \epsilon$ terms in (11) are suppressed. When this is not the case, they generate a large contribution to the variance and, in order to flatten them, the parametrization complementary to (7) is necessary,

$d z=d \beta\left(\frac{d \alpha}{d \beta}+i\right)$

which gives

$$
\begin{aligned}
I_{f}= & -\frac{i \pi}{g_{\epsilon}} \int_{\beta_{-}}^{\beta_{+}} d \beta \\
& \times\left[\left(\frac{\epsilon}{-y_{\beta}}+i\right) \phi\left(-y_{\beta}\right)-\left(\frac{\epsilon}{y_{\beta}}+i\right) \phi\left(y_{\beta}\right)\right],
\end{aligned}
$$

where

$y_{\beta}:=e^{\pi \beta} \sqrt{1-\left(\frac{\epsilon}{e^{\pi \beta}}\right)^{2}}, \quad \beta_{-}=\frac{1}{\pi} \ln \frac{\epsilon}{\sin \epsilon}, \quad \beta_{+}=\frac{\ln \epsilon}{\pi}$.

\footnotetext{
${ }^{1}$ From now on, we omit $\lim _{\epsilon \rightarrow 0}$ and consider $\epsilon$ as an infinitesimal parameter.
} 
Again, (15) is correctly normalized also for a small but not vanishing $\epsilon$. Comparing (12) to (15) gives now

$$
\begin{aligned}
g(y) & =-\frac{g_{\epsilon}}{\ln (\sin \epsilon)} \frac{y}{\left(y^{2}+\epsilon^{2}\right)}, \quad y=e^{\pi \beta} \sqrt{1-\left(\frac{\epsilon}{e^{\pi \beta}}\right)^{2}}, \\
\beta & =\frac{\ln (\epsilon)-\rho \ln (\sin \epsilon)}{\pi} .
\end{aligned}
$$

\section{Multichanneling}

Flattening the whole $1 /(x+i \epsilon)$ behaviour of (9) requires a merging of (13) and (16), whose densities we dub $g_{1}(y)$ and $g_{2}(y)$. This can be achieved via a multichannel approach with combined density $g_{\mathrm{c}}(y):=\alpha_{1} g_{1}(y)+\alpha_{2} g_{2}(y)$ and $\alpha_{1}+\alpha_{2}=1$,

$I_{f}=\int_{0}^{1} d \rho \frac{f(-y)+f(y)}{g_{\mathrm{c}}(y)}$.

In (17), $\rho$ is generated according to the distribution $g_{1,2}(y)$ with probability $\alpha_{1,2}$, and the a-priori weights $\alpha_{1,2}$ can be optimized as described in [14].

To reduce the variance when $\phi(x)$ peaks inside $-1 \leq x \leq$ 1 in a known way, it is also possible to include an arbitrary numbers of further channels $g_{i}(y)(i>2)$. However, care must be taken due to the fact that the MC weight of (12) includes both the $f(-y)$ and $f(y)$ contributions. To determine the corresponding density we observe that

$\int_{0}^{1} d y\left[g_{i}(-y)+g_{i}(y)\right]=\int_{-1}^{1} d x g_{i}(x)$,

which means that if $x$ is randomly chosen in $-1 \leq x \leq 1$, the density is $g_{i}(-|x|)+g_{i}(|x|)$. Hence, the MC weight is $(f(-y)+f(y)) /\left(g_{i}(-y)+g_{i}(y)\right)$, with $g_{i}$ normalized such that

$\int_{-1}^{1} d x g_{i}(x)=1$

In summary, with $N_{\text {ch }}$ channels (including $g_{1}$ and $g_{2}$ ), the more general multichannel MC mapping reads

$I_{f}=\int_{0}^{1} d \rho \frac{f(-y)+f(y)}{g_{\text {tot }}(y)}, d \rho=g_{\text {tot }}(y) d y$,

where

$g_{\text {tot }}(y)=g_{\mathrm{c}}(y)+\sum_{i=3}^{N_{\mathrm{ch}}} \alpha_{i}\left(g_{i}(-y)+g_{i}(y)\right)$,

with arbitrary (but self-adjustable) weights fulfilling $\sum_{i=1}^{N_{\text {ch }}} \alpha_{i}=1$. In the actual MC used to produce the results presented in this paper we superimpose on $g_{\mathrm{c}}(y)$ a flat distribution $g_{3}(x)=1 / 2$ and a channel

$g_{4}(x)=\frac{1}{2 \ln \frac{1+\delta}{\delta}} \frac{1}{1-|x|+\delta}, \quad \delta=10^{-4}$,

which takes care of peaks around $|x|=1$.

\section{Principal value integrals}

It is often useful to deal with improper integrals, whose behaviour at large values of the integration variables is defined via the Cauchy principal value. The fact that the two symmetric points with respect to $x=0$ are always considered together, makes the use of (20) very convenient. As a matter of notation, we define

$f d \sigma:=\lim _{\Lambda \rightarrow \infty} \int_{-\Lambda}^{\Lambda} d \sigma$

which can be mapped onto the interval $[-1,1]$ by changing variable,

$\sigma=\frac{x}{1-x^{2}}$.

Thus, for instance,

$f d \sigma \frac{\phi(\sigma)}{\sigma+i \epsilon}=\int_{-1}^{1} d x \frac{1+x^{2}}{1-x^{2}} \phi\left(\frac{x}{1-x^{2}}\right) \frac{1}{x+i \epsilon}$,

where we understand the symmetric treatment of (20), so that (25) is well defined even when $\phi(\sigma)$ approaches a constant as $\sigma \rightarrow \pm \infty$.

Multiple integrals

Equation (20) can be easily extended to $n$-fold integrals of the type

$I_{f, n}:=\int_{-1}^{1} \prod_{j=1}^{n}\left(d x_{j}\right) f(\{x\})$,

with $f(\{x\})=\phi(\{x\}) / \prod_{j=1}^{n}\left(x_{j}+i \epsilon\right)$. Our notation is such that $\{x\}=x_{1}, x_{2}, \ldots, x_{n}$ and $\phi(\{x\})$ is a smooth function at $\{x\}=\{0\}$. The result is

$I_{f, n}=\int_{0}^{1} \prod_{j=1}^{n}\left(d \rho_{j}\right) \frac{f(-\{y\})+f(\{y\})}{\prod_{j=1}^{n} g_{\text {tot }}\left(y_{j}\right)}$,

where $d \rho_{j}=g_{\text {tot }}\left(y_{j}\right) d y_{j}$ and the numerator stands for a sum over the $2^{n}$ terms with positive or negative arguments. For instance, when $\{y\}=y_{1}, y_{2}$,

$$
\begin{aligned}
& f(-\{y\})+f(\{y\}) \\
& =f\left(-y_{1},-y_{2}\right)+f\left(-y_{1}, y_{2}\right) \\
& \quad+f\left(y_{1},-y_{2}\right)+f\left(y_{1}, y_{2}\right) .
\end{aligned}
$$

Equation (27) can be generalized to more poles per variable, moved away from arbitrary domains $\in \mathbb{R}$, either by partial fractioning the integrand or by splitting the integration region into sub-intervals. However, configurations like that never appear in what follows, so we do not pursue a detailed analysis in this direction. 


\subsection{Method 2}

As an alternative to the above method, one can apply separate changes of variables to flatten the real and imaginary parts of the pole factor(s) in the integrand. Consider the integral

$\mathcal{I}_{1}[\phi]:=\int_{-a}^{a} d x \frac{\phi(x)}{x+i \epsilon}=\int_{0}^{a} d x \frac{x \phi^{-}(x)-i \epsilon \phi^{+}(x)}{x^{2}+\epsilon^{2}}$,

where $\phi^{ \pm}(x)=\phi(x) \pm \phi(-x)$. We can write this as

$$
\begin{aligned}
\mathcal{I}_{1}[\phi] & =\int_{0}^{a / \epsilon} d y \frac{y \phi^{-}(\epsilon y)-i \phi^{+}(\epsilon y)}{1+y^{2}} \\
& =\int_{0}^{r_{m}} d r \phi^{-}\left(\epsilon \sqrt{\mathrm{e}^{2 r}-1}\right)-i \int_{0}^{\theta_{m}} d \theta \phi^{+}(\epsilon \tan \theta),
\end{aligned}
$$

where

$r_{m}:=\ln \left(1+a^{2} / \epsilon^{2}\right) / 2, \quad \theta_{m}:=\arctan (a / \epsilon)$.

Each of these integrals has optimal variance reduction (in the absence of information about $\phi$ ) and is therefore suited to numerical integration as long as $\phi(x)$ is smooth at $x=$ 0 . Note that the $\epsilon /\left(x^{2}+\epsilon^{2}\right)$ and $x /\left(x^{2}+\epsilon^{2}\right)$ behaviours of (29) correspond to the local densities in (13) and (16), respectively.

The method is easily generalised to two variables. Consider

$$
\begin{aligned}
\mathcal{I}_{2}[\phi] & :=\int_{-a}^{a} d x_{1} d x_{2} \frac{\phi\left(x_{1}, x_{2}\right)}{\left(x_{1}+i \epsilon\right)\left(x_{2}+i \epsilon\right)} \\
& =\int_{0}^{a} d x_{1} d x_{2} \frac{\Phi\left(x_{1}, x_{2} ; \epsilon\right)}{\left(x_{1}^{2}+\epsilon^{2}\right)\left(x_{2}^{2}+\epsilon^{2}\right)},
\end{aligned}
$$

where

$\Phi\left(x_{1}, x_{2} ; \epsilon\right)=x_{1} x_{2} \phi^{11}-i \epsilon\left(x_{1} \phi^{10}+x_{2} \phi^{01}\right)-\epsilon^{2} \phi^{00}$

with

$$
\begin{aligned}
& \phi^{00}=\phi\left(x_{1}, x_{2}\right)+\phi\left(-x_{1}, x_{2}\right)+\phi\left(x_{1},-x_{2}\right)+\phi\left(-x_{1},-x_{2}\right), \\
& \phi^{10}=\phi\left(x_{1}, x_{2}\right)-\phi\left(-x_{1}, x_{2}\right)+\phi\left(x_{1},-x_{2}\right)-\phi\left(-x_{1},-x_{2}\right), \\
& \phi^{01}=\phi\left(x_{1}, x_{2}\right)+\phi\left(-x_{1}, x_{2}\right)-\phi\left(x_{1},-x_{2}\right)-\phi\left(-x_{1},-x_{2}\right), \\
& \phi^{11}=\phi\left(x_{1}, x_{2}\right)-\phi\left(-x_{1}, x_{2}\right)-\phi\left(x_{1},-x_{2}\right)+\phi\left(-x_{1},-x_{2}\right) .
\end{aligned}
$$

For MC evaluation, we proceed as follows: for each shot, generate $x_{1 r}, x_{1 t}, x_{2 r}, x_{2 t}$ where

$$
\begin{array}{ll}
x_{1 r}=\epsilon \sqrt{\mathrm{e}^{2 r_{1}}-1}, & x_{1 t}=\epsilon \tan \theta_{1}, \\
x_{2 r}=\epsilon \sqrt{\mathrm{e}^{2 r_{2}}-1}, & x_{2 t}=\epsilon \tan \theta_{2}
\end{array}
$$

where

$0<r_{1,2}<r_{m}, \quad 0<\theta_{1,2}<\theta_{m}$ uniformly, with $r_{m}$ and $\theta_{m}$ as in (31). In (34), set $x_{1}=x_{1 t}$ when the first superscript is 0 and $x_{1}=x_{1 r}$ when it is 1 , and similarly for $x_{2}$ according to the second superscript. The weights for the real and imaginary parts are then

$w_{r}=\phi^{11} r_{m}^{2}-\phi^{00} \theta_{m}^{2}, \quad w_{i}=-\left(\phi^{10}+\phi^{01}\right) r_{m} \theta_{m}$.

The generalisation of (32) to $n$ variables is clear: $\phi^{\left\{k_{j}\right\}}$ has superscript $k_{j}=1$ in the $j$ th location when there is an $x_{j}$ in the integrand, otherwise $k_{j}=0$. The symmetrized function $\Phi$ becomes

$\Phi\left(\left\{x_{j}\right\} ; \epsilon\right)=(-i \epsilon)^{n} \sum_{\left\{k_{j}=0,1\right\}} \prod_{j=1}^{n}\left(i x_{j} / \epsilon\right)^{k_{j}} \phi^{\left\{k_{j}\right\}}\left(\left\{x_{j}\right\}\right)$,

where

$\phi^{\left\{k_{j}\right\}}\left(\left\{x_{j}\right\}\right)=\sum_{\left\{l_{j}=0,1\right\}} \prod_{j=1}^{n}(-1)^{k_{j} l_{j}} \phi\left(\left\{(-1)^{l_{j}} x_{j}\right\}\right)$.

For MC evaluation, for each shot, generate two points in the $n$-dimensional hypercube

$x_{j r}=\epsilon \sqrt{\mathrm{e}^{2 r_{j}}-1}, \quad x_{j t}=\epsilon \tan \theta_{j}$,

where again $0<r_{j}<r_{m}$ and $0<\theta_{j}<\theta_{m}$ uniformly. In (39), set $x_{j}=x_{j t}$ when $k_{j}=0$ and $x_{j}=x_{j r}$ when $k_{j}=1$. The weight is then

$w_{r}+i w_{i}=\left(-i \theta_{m}\right)^{n} \sum_{\left\{k_{j}=0,1\right\}} \prod_{j=1}^{n}\left(i r_{m} / \theta_{m}\right)^{k_{j}} \phi^{\left\{k_{j}\right\}}\left(\left\{x_{j}\right\}\right)$.

Note that each shot involves $2 n$ random numbers for $\left\{x_{j t}\right\}$ and $\left\{x_{j r}\right\}$, and then $4^{n}$ function evaluations at $x_{j}= \pm x_{j t}$ and $\pm x_{j r}$, so the computation time increases rapidly with the number of variables.

\subsection{Choosing $\epsilon$}

Here we perform a study of the value of $\epsilon$ to be used in practice. More specifically, we compare the numerical and analytic determinations of the three-fold test integral

$$
\begin{aligned}
T(\epsilon) & =\int_{-1}^{1} \prod_{j=1}^{3}\left(\frac{d x_{j}}{x_{j}+i \epsilon}\right) \sum_{j_{1}, j_{2}, j_{3}=0}^{1} x_{1}^{j_{1}} x_{2}^{j_{2}} x_{3}^{j_{3}} \\
& =\left(8-6 \pi^{2}\right)+i \pi\left(\pi^{2}-12\right),
\end{aligned}
$$

whose behaviour at $x_{j} \sim 0$ mimics a typical multidimensional environment. The result of this comparison is given in Fig. 1, where the solid (dashed) line represents the real (imaginary) part of (43). Bullets and squares with errors are the MC predictions for $\Re e[T(\epsilon)]$ and $\Im m[T(\epsilon)]$, respectively. To quantify the effect of a nonzero $\epsilon$ on the MC esti- 


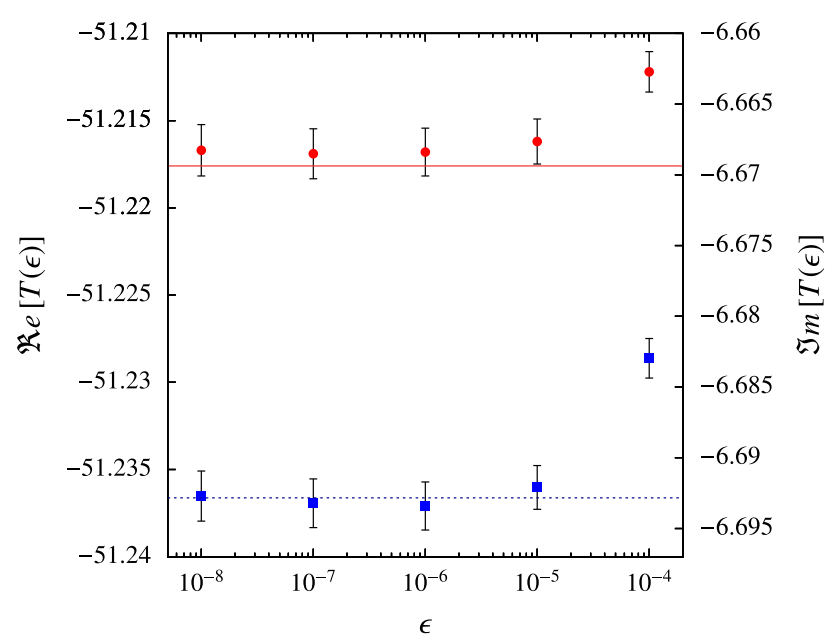

Fig. 1 MC results for the real (red bullets) and imaginary (blue squares) part of (42). They have been obtained with $10^{10} \mathrm{MC}$ shots per point, corresponding to $8 \times 10^{10}$ calls to the integrand. To minimize the statistical fluctuations, the same sequence of random numbers is used for all values of $\epsilon$

mate $Q_{\mathrm{MC}}(\epsilon) \pm \Delta Q(\epsilon)$ of a known quantity $Q$, it is convenient to introduce the estimators

$\Delta_{1}(\epsilon)=\frac{\left|Q-Q_{\mathrm{MC}}(\epsilon)\right|}{|Q|}, \Delta_{2}(\epsilon)=\max \left(\epsilon, \frac{\Delta Q(\epsilon)}{|Q|}\right)$.

Requiring the $\epsilon \neq 0$ bias on $Q_{\mathrm{MC}}(\epsilon)$ to be of the order of the maximum between $\epsilon$ and the relative MC error gives the condition

$R(\epsilon, Q):=\frac{\Delta_{1}(\epsilon)}{\Delta_{2}(\epsilon)} \sim \mathcal{O}(1)$.

Table 1 reports the $R$ value of the entries of Fig. 1 and their MC accuracy defined as

$\delta(\epsilon):=\frac{\max (\Delta \Re e[T(\epsilon)], \Delta \Im m[T(\epsilon)])}{\left|T_{\mathrm{MC}}(\epsilon)\right|}$.

From Fig. 1 and Table 1 we infer that a range $10^{-8} \leq \epsilon \leq$ $10^{-6}$ is adequate to achieve MC estimates accurate at the level of three parts in $10^{5}$. Since the results presented in this paper are never more accurate than this, we set, for definiteness, $\epsilon=10^{-7}$. However, the last row of Table 1 shows that numerically stable predictions are produced also with a smaller $\epsilon$ and a larger MC statistics. From this, we deduce that the $\epsilon \neq 0$ bias can be reduced to be negligible in most practical applications, and that the accuracy of our method is driven by the $\mathrm{MC}$ error.

\section{A semi-numerical integration algorithm for MIs}

In this section we illustrate how the approach of Sect. 2 can be successfully applied to produce stable and precise seminumerical MC estimates of loop MIs. This is achieved in
Table 1 The ratio in (45) for the real and imaginary parts of $T(\epsilon)$ in (42) computed with $10^{10}\left(3 \times 10^{11}\right)$ MC shots when $\epsilon \geq 10^{-8}\left(\epsilon=10^{-12}\right)$. The last column reports the MC accuracy defined in (46)

\begin{tabular}{llll}
\hline$\epsilon$ & $R(\epsilon, \Re e[T])$ & $R(\epsilon, \Im m[T])$ & $\delta(\epsilon)$ \\
\hline $10^{-4}$ & 1.1 & 7.0 & $3 \times 10^{-5}$ \\
$10^{-5}$ & 1.1 & 0.5 & $3 \times 10^{-5}$ \\
$10^{-6}$ & 0.6 & 0.3 & $3 \times 10^{-5}$ \\
$10^{-7}$ & 0.5 & 0.2 & $3 \times 10^{-5}$ \\
$10^{-8}$ & 0.6 & 0.1 & $3 \times 10^{-5}$ \\
$10^{-12}$ & 0.02 & 1.5 & $7 \times 10^{-6}$ \\
\hline
\end{tabular}



Fig. 2 The scalar three-point one-loop function with arbitrary kinematics and masses $C\left(P^{2}, p_{1}^{2}, p_{2}^{2}, m_{0}, m_{1}, m_{2}\right)$

two steps. Firstly, we integrate analytically over the energy components of the loop momenta, which is always doable by means of the Cauchy integral theorem. In addition, depending on the case at hand, some of the loop angular integrals can also be performed analytically. In this way, integral representations of MIs can be easily obtained. Secondly, we give up any attempt towards a fully analytic integration, which may be difficult, and integrate numerically over the leftover loop components. The integrand to be evaluated is usually plagued by threshold singularities. Single poles migrate towards the real integration domain for some kinematic configurations, so that a blind numerical integration over denominators deformed by the Feynman $i \epsilon$ prescription gives large errors. However, this is precisely the situation for which our approach is designed. We mitigate these problems by retaining a finite small value of $\epsilon$, flattening the real and imaginary parts of pole contributions, and applying multichannel mappings. ${ }^{2}$ In what follows we illustrate the performance of this strategy by means of two examples.

\footnotetext{
2 The use of threshold counterterms [15] could further improve the precision of our approach.
} 
Table 2 Numerical estimates of the one-loop integral (52) multiplied by $m^{2}$, compared to the analytic result of [16].

Numbers obtained with $2 \times 10^{7}$

$\mathrm{MC}$ points. The MC errors are indicated between parentheses

\begin{tabular}{lll}
\hline$\sqrt{\tau}$ & MC result & Analytic result \\
\hline 0.01 & $9.85(2) \times 10^{-7}-i 4.9408(92)$ & $0-i 4.9348$ \\
0.2 & $9.90(1) \times 10^{-7}-i 4.9482(55)$ & $0-i 4.9513$ \\
0.5 & $1.0233(8) \times 10^{-6}-i 5.0361(41)$ & $0-i 5.0412$ \\
1.99 & $1.4341(9) \times 10^{-5}-i 1.0783(3) \times 10^{1}$ & $0-i 1.0782 \times 10^{1}$ \\
2.01 & $1.5350(6)-i 1.2006(3) \times 10^{1}$ & $1.5343-i 1.2006 \times 10^{1}$ \\
10 & $1.4216(3)+i 5.5007(30) \times 10^{-1}$ & $1.4216+i 5.5030 \times 10^{-1}$ \\
$10^{2}$ & $2.8562(8) \times 10^{-2}+i 3.6999(15) \times 10^{-2}$ & $2.8557 \times 10^{-2}+i 3.6990 \times 10^{-2}$ \\
$10^{4}$ & $5.7141(20) \times 10^{-6}+i 1.6258(5) \times 10^{-5}$ & $5.7116 \times 10^{-6}+i 1.6258 \times 10^{-5}$ \\
\hline
\end{tabular}

\subsection{A one-loop example}

Consider the three-point function of Fig. 2 in the case $m_{0}=$ $m_{1}=m_{2}=m, p_{1}^{2}=p_{2}^{2}=0$ and timelike $P$. Rescaling all momenta by $m$

$$
\begin{aligned}
& \frac{q}{m}=\left(t, \rho c_{\theta}, \rho s_{\theta} s_{\phi}, \rho s_{\theta} c_{\phi}\right), \frac{P}{m}=(\sqrt{\tau}, 0,0,0), \\
& \frac{p_{1}}{m}=\frac{\sqrt{\tau}}{2}(1,1,0,0), \frac{p_{2}}{m}=\frac{\sqrt{\tau}}{2}(1,-1,0,0),
\end{aligned}
$$

gives

$$
\begin{aligned}
C_{0}:= & C\left(P^{2}, 0,0, m, m, m\right)=\frac{2 \pi}{m^{2}} \int_{-1}^{1} d c_{\theta} \int_{0}^{\infty} d \rho \rho^{2} \\
& \times \int_{-\infty}^{\infty} d t \frac{1}{\left(\sigma_{0}+i \epsilon\right)\left(\sigma_{1}+i \epsilon\right)\left(\sigma_{2}+i \epsilon\right)},
\end{aligned}
$$

with

$\sigma_{0}=\frac{q^{2}}{m^{2}}-1, \sigma_{1}=\frac{(q-P)^{2}}{m^{2}}-1, \sigma_{2}=\frac{\left(q-p_{2}\right)^{2}}{m^{2}}-1$.

One splits

$$
\begin{aligned}
\frac{1}{\sigma_{2}+i \epsilon}= & \frac{1}{2 R_{2}}\left(\frac{1}{t-\sqrt{\tau} / 2-R_{2}+i \epsilon}\right. \\
& \left.-\frac{1}{t-\sqrt{\tau} / 2+R_{2}-i \epsilon}\right)
\end{aligned}
$$

with $R_{2}^{2}:=\rho^{2}+\frac{\tau}{4}+\sqrt{\tau} \rho c_{\theta}+1$. Thus

$$
\begin{aligned}
\int_{-1}^{1} d c_{\theta} \frac{1}{\sigma_{2}+i \epsilon}= & \frac{1}{\sqrt{\tau} \rho}\left(\ln \frac{t-\sqrt{\tau} / 2-R_{2}^{-}+i \epsilon}{t-\sqrt{\tau} / 2-R_{2}^{+}+i \epsilon}\right. \\
& \left.+\ln \frac{t-\sqrt{\tau} / 2+R_{2}^{-}-i \epsilon}{t-\sqrt{\tau} / 2+R_{2}^{+}-i \epsilon}\right),
\end{aligned}
$$

where $R_{2}^{ \pm}:=\sqrt{(\sqrt{\tau} / 2 \pm \rho)^{2}+1}$. The cut of the logarithms with $+i \epsilon(-i \epsilon)$ is in the lower (upper) $t$ complex half-plane, so that the integration over $t$ in (49) is trivial once one rewrites

$$
\frac{1}{\left(\sigma_{0}+i \epsilon\right)\left(\sigma_{1}+i \epsilon\right)}=\frac{1}{4 R_{0}^{2}}\left(\frac{1}{t-R_{0}+i \epsilon}-\frac{1}{t+R_{0}-i \epsilon}\right)
$$

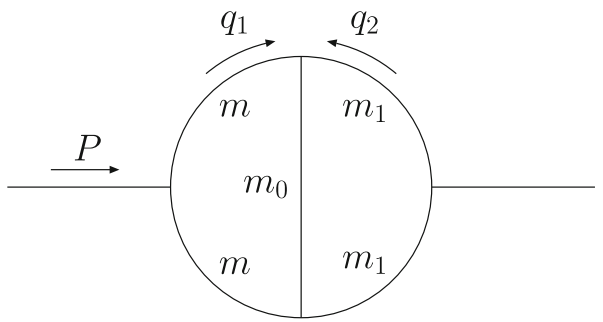

Fig. 3 The two-loop self-energy diagram $S_{2}\left(m, m_{0}, m_{1}\right)$

$$
\times\left(\frac{1}{t-\sqrt{\tau}-R_{0}+i \epsilon}-\frac{1}{t-\sqrt{\tau}+R_{0}-i \epsilon}\right),
$$

with $R_{0}^{2}:=\rho^{2}+1$. The results is

$$
\begin{aligned}
C_{0}= & \frac{2 i \pi^{2}}{m^{2} \tau} \int_{-\infty}^{\infty} \frac{d r}{r-i \epsilon}\left[\theta\left(r+\frac{\sqrt{\tau}}{2}-1\right) L\left(r+\frac{\sqrt{\tau}}{2}, \sqrt{\tau}\right)\right. \\
& \left.+\theta\left(r-\frac{\sqrt{\tau}}{2}-1\right) L\left(r-\frac{\sqrt{\tau}}{2},-\sqrt{\tau}\right)\right],
\end{aligned}
$$

where

$$
L(r, \sqrt{\tau}):=\ln \frac{r-\frac{\sqrt{\tau}}{2}+R(r,-\sqrt{\tau})-i \epsilon}{r-\frac{\sqrt{\tau}}{2}+R(r, \sqrt{\tau})-i \epsilon}
$$

and

$$
R(r, \sqrt{\tau}):=\sqrt{\tau / 4+r^{2}+\sqrt{\tau} \sqrt{r^{2}-1}} .
$$

When $\sqrt{\tau}>2$, the first integrand of (52) develops a pole at $r=i \epsilon$ that migrates towards the integration region in the limit $\epsilon \rightarrow 0$. Treating this with the strategy of Sect. 2 gives the results presented in Table 2 .

\subsection{A two-loop example}

We study the two-loop self-energy scalar diagram of Fig. 3 .

For a timelike $P / m=(\sqrt{\tau}, \mathbf{0})$ and $m_{1}=m$ it reads

$$
S_{2}:=S_{2}\left(m, m_{0}, m\right)=\frac{1}{m^{2}} \int d^{4} \omega_{1} d^{4} \omega_{2} \prod_{j=1}^{5} \frac{1}{\sigma_{j}+i \epsilon},
$$


where

$\omega_{i}:=q_{i} / m=\left(t_{i}, \rho_{i}\right)$

and

$$
\begin{aligned}
& \sigma_{1}=\omega_{1}^{2}-1, \sigma_{2}=\omega_{2}^{2}-1, \\
& \sigma_{3}=\sigma_{1}+\tau-2 \sqrt{\tau} t_{1}, \sigma_{4}=\sigma_{2}+\tau+2 \sqrt{\tau} t_{2}, \\
& \sigma_{5}=\left(t_{1}+t_{2}\right)^{2}-\rho_{1}^{2}-\rho_{2}^{2}-2 \rho_{1} \rho_{2} c_{\theta}-\mu_{0},
\end{aligned}
$$

with $\mu_{0}:=m_{0}^{2} / m^{2}$. Integrating over the angular variables gives

$$
\begin{aligned}
S_{2}= & \frac{4 \pi^{2}}{m^{2}} \int_{0}^{\infty} d \rho_{1} \rho_{1} \int_{0}^{\infty} d \rho_{2} \rho_{2} \int d t_{1} d t_{2} \prod_{j=1}^{4} \frac{1}{\sigma_{j}+i \epsilon} \\
& \times \ln \frac{\left(t_{1}+t_{2}\right)^{2}-\left(\rho_{1}-\rho_{2}\right)^{2}-\mu_{0}+i \epsilon}{\left(t_{1}+t_{2}\right)^{2}-\left(\rho_{1}+\rho_{2}\right)^{2}-\mu_{0}+i \epsilon} .
\end{aligned}
$$

The integration over $t_{1}$ and $t_{2}$ is trivial and produces

$S_{2}=\frac{2 \pi^{4}}{m^{2} \tau} \sum_{\lambda_{1,2}= \pm} \int_{-\infty}^{\infty} d r_{1} \int_{-\infty}^{\infty} d r_{2} \frac{F\left(r_{1}, r_{2}, \lambda_{1}, \lambda_{2}\right)}{\left(r_{1}-i \epsilon\right)\left(r_{2}-i \epsilon\right)}$

where

$$
\begin{aligned}
& F\left(r_{1}, r_{2}, \lambda_{1}, \lambda_{2}\right)=\lambda_{1} \lambda_{2} \theta\left(A_{1}-1\right) \theta\left(A_{2}-1\right) \\
& \times \ln \frac{r_{1}+r_{2}+\sqrt{\left(\sqrt{A_{1}^{2}-1}+\sqrt{A_{2}^{2}-1}\right)^{2}+\mu_{0}}-i \epsilon}{r_{1}+r_{2}+\sqrt{\left(\sqrt{A_{1}^{2}-1}-\sqrt{A_{2}^{2}-1}\right)^{2}+\mu_{0}}-i \epsilon}
\end{aligned}
$$

and $A_{i}:=r_{i}+\lambda_{i} \sqrt{\tau} / 2$. Threshold singularities at $r_{1,2}=i \epsilon$ are present when $\lambda_{1,2}=+1$ if $\sqrt{\tau}>2$. When $m_{0}=0$, a two-dimensional implementation of the method of Sect. 2 gives the results reported in Table 3. Larger MC errors correspond to smaller values of $\rho$. However, we observe that when $\mu_{0} \neq 0$ this effect is mitigated. For instance, a $10^{9} \mathrm{MC}$-point estimate with $\rho=.1$ gives

$-\frac{m^{2} \tau}{\pi^{4}} S_{2}(m, m, m)=8.582(6)-i 2.706(4)$.

\section{Gluing together lower-loop structures}

Here we show how higher-loop integrals can be expressed in terms of lower-loop building blocks. Throughout this section dimensionful quantities are rescaled by an arbitrary mass $m$, so that loop momenta are written as in (54) and, in particular,

$\omega:=q / m=\left(t, \rho c_{\theta}, \rho s_{\theta} s_{\phi}, \rho s_{\theta} c_{\phi}\right)$.

Furthermore, we define

$$
\begin{aligned}
\mu_{i} & :=m_{i}^{2} / m^{2}, \quad \tau:=P^{2} / m^{2}, \chi:=\left(p_{2}-p_{3}\right)^{2} / m^{2}, \\
\tau_{i} & :=p_{i}^{2} / m^{2}, \quad \tau_{i j}:=\tau_{i}-\tau_{j}, \\
\lambda_{i j} & :=\lambda\left(\tau, \tau_{i}, \tau_{j}\right),
\end{aligned}
$$

Table 3 The two-loop integral (57) with $m_{0}=0$ multiplied by $-m^{2} \tau / \pi^{4}$ for several values of $\rho:=4 / \tau$. Numbers obtained with $10^{9}\left(10^{10}\right)$ MC points when $\rho>1(\rho<1)$. The analytic result is taken from [17]. MC errors between parentheses

\begin{tabular}{lll}
\hline$\rho$ & MC result & Analytic result \\
\hline 0.1 & $8.49(1)-i 1.94(2)$ & $8.495-i 1.927$ \\
0.3 & $9.34(1)-i 5.47(2)$ & $9.340-i 5.460$ \\
0.5 & $9.19(1)-i 9.71(1)$ & $9.195-i 9.716$ \\
0.7 & $7.39(1)-i 15.79(1)$ & $7.396-i 15.783$ \\
0.9 & $-1.03(2)-i 27.591(8)$ & $-1.061-i 27.581$ \\
1.1 & $-15.538(2)-i 1.8314(4) \times 10^{-5}$ & $-15.540+i 0$ \\
1.3 & $-7.9915(8)-i 5.1218(7) \times 10^{-6}$ & $-7.9921+i 0$ \\
1.5 & $-5.5608(6)-i 2.9000(4) \times 10^{-6}$ & $-5.5614+i 0$ \\
1.7 & $-4.2990(5)-i 2.0139(3) \times 10^{-6}$ & $-4.2996+i 0$ \\
1.9 & $-3.5153(5)-i 1.5412(2) \times 10^{-6}$ & $-3.5157+i 0$ \\
\hline
\end{tabular}

$$
\begin{aligned}
k^{2} & :=\lambda_{12} / \tau^{2}, \quad k_{ \pm}:=\sqrt{k^{2} \pm i \epsilon}, \\
\left(k^{\prime}\right)^{2} & :=\lambda_{34} / \tau^{2},
\end{aligned}
$$

and study cases up to a $P:=p_{1}+p_{2} \rightarrow p_{3}+p_{4}$ kinematics of the form

$$
\begin{aligned}
& p_{1}=\frac{m}{2 \sqrt{\tau}}\left(\tau+\tau_{12}, \lambda_{12}^{\frac{1}{2}}, 0,0\right), \\
& p_{2}=\frac{m}{2 \sqrt{\tau}}\left(\tau-\tau_{12},-\lambda_{12}^{\frac{1}{2}}, 0,0\right), \\
& p_{3}=\frac{m}{2 \sqrt{\tau}}\left(\tau+\tau_{34}, \lambda_{34}^{\frac{1}{2}} \cos \theta_{13}, \lambda_{34}^{\frac{1}{2}} \sin \theta_{13}, 0\right), \\
& p_{4}=\frac{m}{2 \sqrt{\tau}}\left(\tau-\tau_{34},-\lambda_{34}^{\frac{1}{2}} \cos \theta_{13},-\lambda_{34}^{\frac{1}{2}} \sin \theta_{13}, 0\right) .
\end{aligned}
$$

Rescaled propagators belonging to the loop momentum $q$ are denoted by

$\sigma_{0}:=q^{2} / m^{2}-\mu_{0}, \quad \sigma_{1}:=(q-P)^{2} / m^{2}-\mu_{1}$,

$\sigma_{2}:=\left(q-p_{2}\right)^{2} / m^{2}-\mu_{2}, \sigma_{3}:=\left(q-p_{3}\right)^{2} / m^{2}-\mu_{3}$.

In addition, we define

$\lambda:=\lambda\left(\tau, \sigma_{0}+\mu_{0}, \sigma_{1}+\mu_{1}\right)$,

and $^{3}$

$$
\begin{aligned}
\sigma_{a}^{i j}:= & \tau_{1}-\tau+\sigma_{j}+\mu_{j}+\frac{k c_{\theta}}{2} \lambda^{\frac{1}{2}}\left(\tau, \sigma_{i}+\mu_{i}, \sigma_{j}+\mu_{j}\right) \\
& +\frac{1}{2}\left(1-\tau_{12} / \tau\right)\left(\tau+\sigma_{i}+\mu_{i}-\sigma_{j}-\mu_{j}\right)
\end{aligned}
$$

$\sigma_{b}^{i j}:=\sigma_{i}+\mu_{i}+\sigma_{j}+\mu_{j}+\tau_{1}+\tau_{2}-\tau-\sigma_{a}^{i j}$,

$\sigma_{c}^{i j}:=\tau_{3}+\sigma_{i}+\mu_{i}+\frac{k^{\prime}}{2} \lambda^{\frac{1}{2}}\left(\tau, \sigma_{i}+\mu_{i}, \sigma_{j}+\mu_{j}\right)$

$3 \sigma_{a, b, c, d}^{i j}$ are the invariants $\left(q-p_{1,2,3,4}\right)^{2} / m^{2}$ computed at values of $t$ and $\rho$ satisfying the conditions $t^{2}-\rho^{2}=\sigma_{i}+\mu_{i}$ and $(t-\sqrt{\tau})^{2}-\rho^{2}=$ $\sigma_{j}+\mu_{j}$. 


$$
\begin{aligned}
& \times\left(c_{\theta} \cos \theta_{13}+s_{\theta} s_{\phi} \sin \theta_{13}\right) \\
& -\frac{1}{2}\left(1+\tau_{34} / \tau\right)\left(\tau+\sigma_{i}+\mu_{i}-\sigma_{j}-\mu_{j}\right), \\
\sigma_{d}^{i j}:= & \sigma_{i}+\mu_{i}+\sigma_{j}+\mu_{j}+\tau_{3}+\tau_{4}-\tau-\sigma_{c}^{i j} .
\end{aligned}
$$

The essence of the procedure is to use $\sigma_{0}$ and $\sigma_{1}$ as integration variables of the method of Sect. 2. This is achieved by multiplying the integrand by

$1=f d \sigma_{0} f d \sigma_{1} \Delta\left(\sigma_{0}, \sigma_{1}, \rho, t\right)$,

where

$$
\begin{aligned}
& \Delta\left(\sigma_{0}, \sigma_{1}, \rho, t\right):=\delta\left(\sigma_{0}+\mu_{0}+\rho^{2}-t^{2}\right) \\
& \quad \times \delta\left(\sigma_{1}-\sigma_{0}+\mu_{1}-\mu_{0}-\tau+2 \sqrt{\tau} t\right) .
\end{aligned}
$$

This gives rise to the appearance of the following three functionals,

$$
\begin{aligned}
\Phi_{1}^{\left(\mu_{0}, \mu_{1}\right)}\left[J_{1}\right] & :=\int d^{4} \omega J_{1} \Delta\left(\sigma_{0}, \sigma_{1}, \rho, t\right), \\
\Phi_{j}^{\left(\mu_{0}, \mu_{1}, \mu_{j}\right)}\left[J_{j}\right] & :=\int d^{4} \omega \frac{J_{j}}{\sigma_{j}+i \epsilon} \Delta\left(\sigma_{0}, \sigma_{1}, \rho, t\right),
\end{aligned}
$$

where $j=2,3$. Assuming $J_{1}$ independent of any angular variable and $J_{2}\left(J_{3}\right)$ independent of $\theta(\phi)$ allows one to compute the functionals once for all. $\Phi_{1}$ reads

$\Phi_{1}^{\left(\mu_{0}, \mu_{1}\right)}\left[J_{1}\right]=\frac{\pi}{2 \tau} \lambda^{\frac{1}{2}} \theta(\lambda) J_{1}$.

As for $\Phi_{2}$, one has

$$
\begin{aligned}
\Phi_{2}^{\left(\mu_{0}, \mu_{1} \mu_{2}\right)}\left[J_{2}\right]= & \frac{1}{4 \tau} \theta(\lambda)\left[\frac{1}{k_{+}} \ln \left(1+\frac{k_{+} \lambda^{\frac{1}{2}}}{A_{2}+i \epsilon}\right)\right. \\
& \left.-\frac{1}{k_{-}} \ln \left(1-\frac{k_{-} \lambda^{\frac{1}{2}}}{A_{2}+i \epsilon}\right)\right] \int_{0}^{2 \pi} d \phi J_{2} .
\end{aligned}
$$

with

$$
\begin{aligned}
A_{2}:= & \left(\sigma_{0}+\mu_{0}\right)\left(1+\frac{\tau_{12}}{\tau}\right)+\left(\sigma_{1}+\mu_{1}\right)\left(1-\frac{\tau_{12}}{\tau}\right) \\
& +\tau_{1}+\tau_{2}-\tau-2 \mu_{2} .
\end{aligned}
$$

Note that (66) and (67) have been analytically continued to configurations with any sign of $\tau, \tau_{1}, \tau_{2}, \lambda_{12}$. Finally

$$
\Phi_{3}^{\left(\mu_{0}, \mu_{1}, \mu_{3}\right)}\left[J_{3}\right]=\frac{\pi}{2 \tau} \lambda^{\frac{1}{2}} \theta(\lambda) \int_{-1}^{1} d c_{\theta} \frac{J_{3}}{A_{3}} \frac{1}{\sqrt{1-\frac{B_{3}}{A_{3}^{2}}}}
$$

in which

$$
\begin{aligned}
A_{3}:= & \left(\sigma_{0}+\mu_{0}\right)\left(1-\frac{\tau_{34}}{\tau}\right)+\left(\sigma_{1}+\mu_{1}\right)\left(1+\frac{\tau_{34}}{\tau}\right) \\
& +\tau_{3}+\tau_{4}-\tau-2 \mu_{3}+\lambda^{\frac{1}{2}} k^{\prime} c_{\theta} \cos \theta_{13}+i \epsilon, \\
B_{3}:= & \frac{\lambda \lambda_{34}}{\tau^{2}} s_{\theta}^{2} \sin ^{2} \theta_{13} .
\end{aligned}
$$

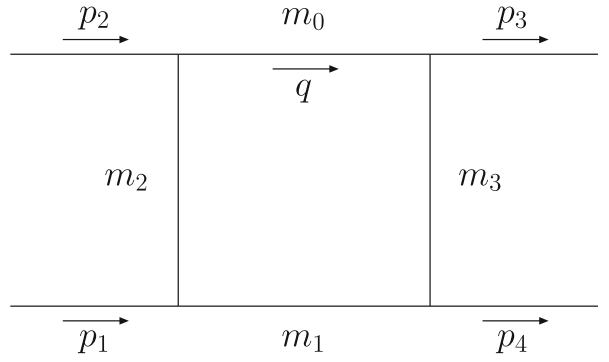

Fig. 4 The scalar four-point one-loop function $D\left(p_{1}^{2}, p_{2}^{2}, p_{3}^{2}, p_{4}^{2},\left(p_{1}+p_{2}\right)^{2},\left(p_{2}-p_{3}\right)^{2}, m_{1}^{2}, m_{2}^{2}, m_{0}^{2}, m_{3}^{2}\right)$ with arbitrary kinematics and masses

\subsection{One-loop examples}

To elucidate the procedure, we first consider gluing tree-level structures to compute the three-point function of Fig. 2-with arbitrary kinematics and masses-and the box diagram of Fig. 4 with $p_{i}^{2}=0$ and $m_{0}=m_{1}=m_{2}=m_{3}=m$, which we dub $C$ and $D_{0}$, respectively. Equations (63), (67) and (69) produce

$m^{2} C=f \prod_{j=0}^{1}\left(\frac{d \sigma_{j}}{\sigma_{j}+i \epsilon}\right) \Phi_{2}^{\left(\mu_{0}, \mu_{1}\right)}[1]$

$m^{4} D_{0}=f \prod_{j=0}^{1}\left(\frac{d \sigma_{j}}{\sigma_{j}+i \epsilon}\right) \Phi_{3}^{\left(\mu_{0}, \mu_{1}, \mu_{3}\right)}\left[\frac{1}{\sigma_{2}+i \epsilon}\right]$.

In (71) the integration over the azimuth angle $\phi$ is trivial,

$\Phi_{2}^{\left(\mu_{0}, \mu_{1}\right)}[1]=\frac{\pi}{2 \tau} \theta(\lambda) \sum_{j= \pm}\left[\frac{1}{j k_{j}} \ln \left(1+\frac{j k_{j} \lambda^{\frac{1}{2}}}{A_{2}+i \epsilon}\right)\right]$

while the integral over $c_{\theta}$ in (72) has to be dealt with numerically using the method of Sect. 2 , which gives

$$
\begin{aligned}
\Phi_{3}^{\left(\mu_{0}, \mu_{1}, \mu_{3}\right)}\left[\frac{1}{\sigma_{2}+i \epsilon}\right]= & \frac{\pi}{\tau} \theta\left(\lambda_{0}\right) f \frac{d \sigma_{2}}{\sigma_{2}+i \epsilon} \\
& \times \frac{\theta\left(\sigma_{2}-\sigma_{2}^{-}\right) \theta\left(\sigma_{2}^{+}-\sigma_{2}\right)}{A_{0} \sqrt{1-\frac{B_{0}}{A_{0}^{2}}}},
\end{aligned}
$$

where

$$
\begin{aligned}
\sigma_{2}^{ \pm} & =\frac{\sigma_{\tau} \pm \lambda_{0}^{\frac{1}{2}}}{2}, \sigma_{\tau}:=\sigma_{0}+\sigma_{1}-\tau, \\
A_{0} & =2\left[\sigma_{2}-\frac{\chi}{\tau}\left(\sigma_{\tau}-2 \sigma_{2}\right)\right]+i \epsilon, \\
B_{0} & =-16 \frac{\chi}{\tau}\left(1+\frac{\chi}{\tau}\right)\left[\sigma_{2}\left(\sigma_{\tau}-\sigma_{2}\right)-\tau-\sigma_{0} \sigma_{1}\right], \\
\lambda_{0} & =\lambda\left(\tau, \sigma_{0}+1, \sigma_{1}+1\right) .
\end{aligned}
$$




\section{Improving the numerical accuracy}

When inserted in (71) and (72), Eqs. (73) and (74) could potentially produce inaccurate results when strong cancellations are expected among different integration regions. This happens if

(a) the integrands do not vanish fast enough at large values of $\sigma_{0,1}$;

(b) $\tau$ is small.

Note that case (a) is relevant to $C$ but not to $D_{0}$, since

$$
\begin{aligned}
& \lim _{\sigma_{0,1} \rightarrow \infty} \Phi_{2}^{\left(\mu_{0}, \mu_{1}\right)}[1] \sim \text { constant } \\
& \lim _{\sigma_{0,1} \rightarrow \infty} \Phi_{3}^{\left(\mu_{0}, \mu_{1}, \mu_{3}\right)}\left[\frac{1}{\sigma_{2}+i \epsilon}\right] \sim \frac{1}{\sigma_{0,1}},
\end{aligned}
$$

while (b) applies to both $C$ and $D_{0}$ due to the common $1 / \tau$ prefactor. In the following paragraphs we illustrate how numerical inaccuracies caused by the configurations (a) and (b) in (76) can be circumvented.

As for case (a), a preliminary analysis is in order to understand the mechanism that makes $C$ finite despite (77). ${ }^{4} \mathrm{We}$ define

$\sigma_{10}:=\sigma_{1}-\sigma_{0}$

in terms of which

$m^{2} C=\lim _{\Lambda \rightarrow \infty} \int_{-\Lambda}^{\Lambda} d \sigma_{10} \int d \sigma_{0} \frac{\Phi_{2}^{\left(\mu_{0}, \mu_{1}\right)}[1]}{\left(\sigma_{0}+i \epsilon\right)\left(\sigma_{0}+\sigma_{10}+i \epsilon\right)}$.

Now the $\sigma_{0}$ integral is convergent by power counting and $\lambda$ in (61) behaves as

$\lim _{\sigma_{10} \rightarrow \infty} \lambda \sim \tilde{\lambda}:=\left(\sigma_{10}+\beta\right)^{2}-\alpha$,

where $\alpha$ and $\beta$ are constants. Replacing $\lambda$ with $\tilde{\lambda}$ in (73) produces an integrand in which all branch points and poles are located in the lower $\sigma_{0}$ complex half-plane. As a result, the integral over $\sigma_{0}$ approaches zero when $\sigma_{10} \rightarrow \pm \infty$, so that the $\Lambda \rightarrow \infty$ limit exists. This same reasoning allows one to construct a class of vanishing integrals defined as

$m^{2} \tilde{C}\left(\alpha, \beta, \Lambda_{0}\right):=\int \frac{d^{4} \omega \theta\left(\left|\sigma_{10}\right|-\Lambda_{0}\right)}{\left(\sigma_{0}+i \epsilon\right)\left(\tilde{\sigma}_{1}+i \epsilon\right)\left(\tilde{\sigma}_{2}+i \epsilon\right)}$,

with

$m^{2} \tilde{\sigma}_{1}:=q^{2}+P^{2}-m_{1}^{2}-2(\tilde{q} \cdot P)$,

$m^{2} \tilde{\sigma}_{2}:=q^{2}+p_{2}^{2}-m_{2}^{2}-2\left(\tilde{q} \cdot p_{2}\right)$,

4 The principal value integrations in (71) are not sufficient to regularize the large $\sigma_{0,1}$ behaviour. In fact, $\Phi_{2}^{\left(\mu_{0}, \mu_{1}\right)}[1]$ approaches two different constants when $\sigma_{0,1} \rightarrow \infty$ or $\sigma_{0,1} \rightarrow-\infty$, so that no cancellation is possible. where $\tilde{q}$ is the asymptotic $\left|\sigma_{10}\right| \rightarrow \infty$ limit of $q$,

$\frac{\tilde{q}}{m}:=(\tilde{t}, \overrightarrow{\tilde{\rho}}), \tilde{t}:=-\frac{\sigma_{10}+\beta}{2 \sqrt{\tau}}, \tilde{\rho}:=\frac{\tilde{\lambda}^{\frac{1}{2}}}{2 \sqrt{\tau}}$.

Again, all cuts and poles lie in the lower $\sigma_{0}$ complex halfplane, so that

$\tilde{C}\left(\alpha, \beta, \Lambda_{0}\right)=0$.

Now $\alpha$ and $\beta$ can be set to obtain a local cancellation of the problematic large $\sigma_{10}$ configurations. ${ }^{5}$ An explicit calculation with $\tau_{1}=\tau_{2}=0$ gives

$\alpha=4 \tau \mu_{2}, \beta=\mu_{1}-\mu_{0}$.

When $\tau_{i} \neq 0$ the accuracy of (71) is improved by the nonvanishing external masses, so that (85) is relevant to this case as well. In summary, the formula

$m^{2} C=m^{2} C-m^{2} \tilde{C}\left(\alpha, \beta, \Lambda_{0}\right)$

produces numerically stable results with $\alpha$ and $\beta$ given in (85) when the same sequence of $\sigma_{0}$ and $\sigma_{1}$ values are used in both $C$ and $\tilde{C}$.

It turns out that the configurations of type (b) of $C$ are also cured by the subtraction in (86). Thus, we are only left with the discussion of the case (b) for $D_{0}$. In the $\tau \rightarrow 0$ region it is convenient to give up the exact formula and use, instead, a few terms of a Taylor expansion in $\tau$ and $\chi$ obtained with the method given in Appendix A,

$$
\begin{aligned}
\frac{D_{0}}{i \pi^{2}}= & \frac{1}{6 m^{4}}\left[1+\frac{\tau+\chi}{10}+\frac{\tau^{2}+\chi^{2}}{70}+\frac{\tau \chi}{140}\right. \\
& \left.+\frac{\tau^{3}+\chi^{3}}{420}+\frac{\tau \chi(\tau+\chi)}{1260}+\mathcal{O}\left(\tau^{4}\right)\right] .
\end{aligned}
$$

By doing that, it is easy to find a value of $\tau$ below which the exact result is well approximated by (87), and above which (72) is accurate.

Results

Here we present the numerical outcome of a MC based on (71), (72), (86) and (87).

The results for $C\left(P^{2}, 0,0, m, m, m\right)$ are shown in Figs. 5 and 6 . In the latter, the relative difference between the $\mathrm{MC}$ and the analytic (AN) non-zero results of the former is plotted in terms of

$\Delta_{R, I}:=1+\left(\frac{\mathrm{MC}-\mathrm{AN}}{\mathrm{AN}}\right)_{R, I}$,

where $R$ and $I$ refer to the real and imaginary parts, respectively. With the given MC statistics, the analytic result is

\footnotetext{
5 Additionally, $\Lambda_{0}$ can be used to control when such a local subtraction has to be performed.
} 




Fig. $5 \mathrm{MC}$ estimates of the real (red bullets) and imaginary (blue squares) part of (86), evaluated with $m_{i}=m, p_{i}^{2}=0$ and $\Lambda_{0}=0$, compared to the analytic output of [16]. Results obtained with $10^{9} \mathrm{MC}$ shots per point

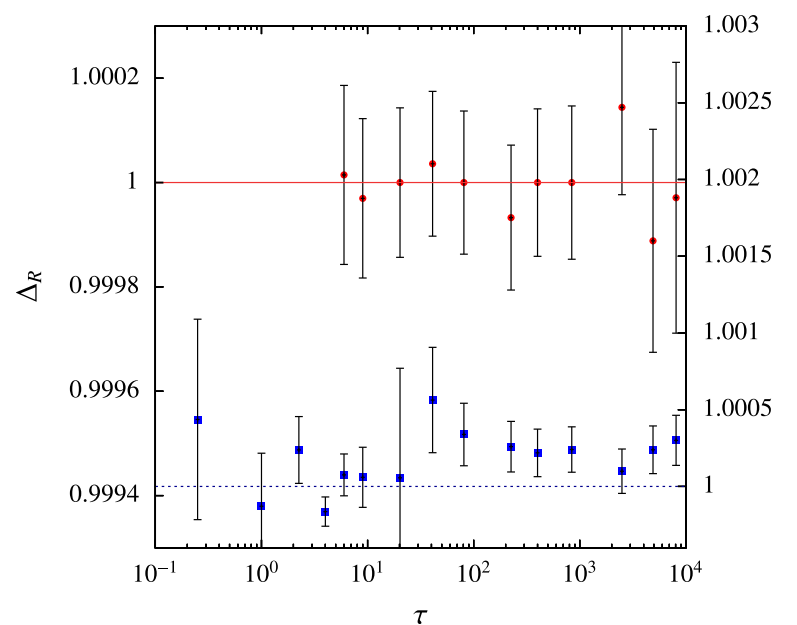

Fig. 6 The relative difference between the MC and the non-zero analytic results of Fig. 5 as defined in (88). Red bullets (blue squares) refer to $\Delta_{R}\left(\Delta_{I}\right)$

reproduced by (86) within a few parts in $10^{4},{ }^{6}$ which is an accuracy comparable to the one of Table 2, but obtained with 50 times more points. The main reason for this difference is that the one-dimensional representation (52) is now replaced by the twofold integration (71). On the other hand, the gluing algorithm is highly modular and can be extended to more complex situations, as we will see in the next subsection.

Table 4 displays our results for $D_{0}$ as a function of $\tau$ and the scattering angle $-\chi / \tau=\left(1+\cos \theta_{13}\right) / 2$. We use the MC evaluation of (72) above $\tau=1$ and the Taylor expansion of (87) when $\tau<1$. In the former case, an accuracy of a few parts in $10^{3}$ is reached with $10^{9} \mathrm{MC}$ points.

${ }^{6}$ Similar results are obtained when $\tau<0$. For instance, with $\tau=-10^{3}$ Eq. (86) gives $m^{2} C=1(1) \times 10^{-6}-i 0.23566(5)$, to be compared to the analytic value $-i 0.23561$.

\subsection{Two and three-loop examples}

\section{Two-loop self-energy}

The two-loop diagram of Fig. 3 can be easily obtained by gluing together a one-loop triangle and a tree-level structure. For instance, with $m_{0}=m_{1}=0$ one has

$$
\begin{aligned}
m^{2} S_{2}(m, 0,0)= & f \prod_{j=0}^{1}\left(\frac{d \sigma_{j}}{\sigma_{j}+i \epsilon}\right) \\
& \times \Phi_{1}^{(1,1)}\left[C\left(\tau, \sigma_{1}+1, \sigma_{0}+1,0,0,0\right)\right] .
\end{aligned}
$$

Equation (89) suffers the inaccuracies of type (a) of (76). To cure this, we use the same strategy described in Sect. 4.1. First, we consider an explicit representation of the triangle as a function of $\sigma_{0}$ and $\sigma_{10}$ in (79),

$C\left(\tau, \sigma_{1}+1, \sigma_{0}+1,0,0,0\right)=2 \frac{i \pi^{2}}{\lambda_{0}^{\frac{1}{2}}} F\left(\sigma_{0}, \sigma_{10}\right)$,

with $\lambda_{0}$ in (75),

$$
\begin{aligned}
F\left(\sigma_{0}, \sigma_{10}\right) & \\
= & \sum_{j=1,2}(-1)^{j}\left\{\operatorname{Li}_{2}\left(\frac{2 \tau}{v_{j}}\right)+\ln \left(\frac{2 \tau}{v_{j}}\right) \ln \left(1-\frac{2 \tau}{v_{j}}\right)\right. \\
& \left.-\frac{1}{4} \ln ^{2}\left(\frac{2 \tau}{v_{j}}-1\right)-\frac{1}{4} \ln ^{2}\left(\frac{u_{j}}{v_{j}}\right)\right\},
\end{aligned}
$$

and

$u_{1}=\tau-\sigma_{10}+\lambda_{0}^{\frac{1}{2}}-i \epsilon \tau, u_{2}=\tau-\sigma_{10}-\lambda_{0}^{\frac{1}{2}}+i \epsilon \tau$

$v_{1}=\tau+\sigma_{10}+\lambda_{0}^{\frac{1}{2}}-i \epsilon \tau, v_{2}=\tau+\sigma_{10}-\lambda_{0}^{\frac{1}{2}}+i \epsilon \tau$.

From this, it is easy to determine a $\left|\sigma_{10}\right| \rightarrow \infty$ asymptotic approximant of (90) that gives zero upon integration over $\sigma_{0,1}$. The results reads ${ }^{7}$

$$
\begin{aligned}
m^{2} \tilde{S}_{2}\left(\Lambda_{c}\right):= & \frac{i \pi^{3}}{\tau} f \prod_{j=0}^{1}\left(\frac{d \sigma_{j}}{\sigma_{j}+i \epsilon}\right) \theta\left(\tilde{\lambda}_{0}\right) \\
& \times \theta\left(\Lambda_{c}^{2}-\frac{\tau^{2}}{\tau^{2}+\sigma_{10}^{2}}\right) \tilde{F}\left(\sigma_{0}, \sigma_{10}\right)=0,
\end{aligned}
$$

where $\tilde{\lambda}_{0}:=\left(\sigma_{10}-\tau\right)^{2}-4 \tau, \Lambda_{c}$ is arbitrary and $\tilde{F}\left(\sigma_{0}, \sigma_{10}\right)$ is constructed by replacing in (91) the $u_{j}, v_{j}$ with their asymptotic counterparts $\tilde{u}_{j}, \tilde{v}_{j}$ defined as

$\tilde{u}_{1}=\tilde{a} \theta\left(\sigma_{10}\right)+\tilde{b} \theta\left(-\sigma_{10}\right)-i \epsilon \tau$,

$\tilde{u}_{2}=\tilde{b} \theta\left(\sigma_{10}\right)+\tilde{a} \theta\left(-\sigma_{10}\right)+i \epsilon \tau$,

$\tilde{v}_{1}=2 \sigma_{10} \theta\left(\sigma_{10}\right)+\tilde{c} \theta\left(-\sigma_{10}\right)-i \epsilon \tau$,

\footnotetext{
7 As in the case of (84), (92) is proven by observing that all cuts and poles lie in the lower $\sigma_{0}$ complex half-plane.
} 
Table 4 Numerical estimates of $m^{4} D_{0}$ compared to the analytic result of [16]. Equation (72) [(87)] is used if $\tau>1[\tau<1]$. When $\tau>1,10^{9} \mathrm{MC}$ points are used. Statistical errors between parentheses

\begin{tabular}{llll}
\hline$\tau$ & $-\chi / \tau$ & Numerical result & Analytic result \\
\hline 0.8 & 0.1 & 1.7798 & 1.7801 \\
0.8 & 0.5 & 1.7274 & 1.7277 \\
0.8 & 0.9 & 1.6790 & 1.6795 \\
10 & 0.1 & $-2.185(9)+i 2.64(9) \times 10^{-1}$ & $-2.1871+i 2.7316 \times 10^{-1}$ \\
10 & 0.5 & $-1.642(4)+i 4.4(4) \times 10^{-2}$ & $-1.6423+i 4.2586 \times 10^{-2}$ \\
10 & 0.9 & $-1.339(4)-i 3.6(4) \times 10^{-2}$ & $-1.3368-i 4.0162 \times 10^{-2}$ \\
100 & 0.1 & $-1.297(3) \times 10^{-1}-i 1.253(2) \times 10^{-1}$ & $-1.2993 \times 10^{-1}-i 1.2556 \times 10^{-1}$ \\
100 & 0.5 & $-4.761(8) \times 10^{-2}-i 5.176(9) \times 10^{-2}$ & $-4.7572 \times 10^{-2}-i 5.1670 \times 10^{-2}$ \\
100 & 0.9 & $-3.090(8) \times 10^{-2}-i 3.447(8) \times 10^{-2}$ & $-3.0812 \times 10^{-2}-i 3.4615 \times 10^{-2}$ \\
1000 & 0.1 & $-2.803(5) \times 10^{-3}-i 5.277(5) \times 10^{-3}$ & $-2.8153 \times 10^{-3}-i 5.2737 \times 10^{-3}$ \\
1000 & 0.5 & $-7.66(2) \times 10^{-4}-i 1.501(2) \times 10^{-3}$ & $-7.6923 \times 10^{-4}-i 1.4986 \times 10^{-3}$ \\
1000 & 0.9 & $-4.68(2) \times 10^{-4}-i 9.20(2) \times 10^{-4}$ & $-4.6848 \times 10^{-4}-i 9.2226 \times 10^{-4}$ \\
\hline
\end{tabular}

$\tilde{v}_{2}=\tilde{c} \theta\left(\sigma_{10}\right)+2 \sigma_{10} \theta\left(-\sigma_{10}\right)+i \epsilon \tau$,

with

$\tilde{a}:=-\frac{2 \tau}{\sigma_{10}}\left(\sigma_{0}+1\right), \tilde{b}:=2\left(\tau-\sigma_{10}\right)$,

$\tilde{c}:=\frac{2 \tau}{\sigma_{10}}\left(\sigma_{10}+\sigma_{0}+1\right)$.

Finally, in the same spirit as (86), we rewrite

$S_{2}(m, 0,0)=S_{2}(m, 0,0)-\tilde{S}_{2}\left(\Lambda_{c}\right)$,

where we understand a local subtraction of the large $\left|\sigma_{10}\right|$ configurations.

In Table 5 we present our numerical estimates based on (95) with $\Lambda_{c}=1 / 2$. An accuracy of the order of $10^{-3}$ is achieved with $10^{9} \mathrm{MC}$ points. We also studied the stability of (95) at small values of $\tau$. For instance, when $\rho=100$ $(\tau=0.04)$ we obtain

$-m^{2} \frac{\tau}{\pi^{4}} S_{2}(m, 0,0)=-0.2090(2)-i 0.1263(3)$.

Note that determining $\tilde{S}_{2}\left(\Lambda_{c}\right)$ requires an analytic knowledge of the integrand. When this is not possible, an alternative approach is to cut away the problematic configurations in a controlled manner. For instance, discarding in (89) integration points with

$\sqrt{\tau^{2} /\left(\tau^{2}+\sigma_{10}^{2}\right)}<\Lambda$

is expected to produce an error of $\mathcal{O}\left(\Lambda^{2}\right)$. Indeed we checked that, with $\Lambda=0.01$, the fraction of the integral discarded by (97) is always below the errors reported in Table 5.

\section{Three-loop self-energy}

Our next example is the scalar three-loop self-energy of Fig. 7, which is attained by gluing together the two trian-
Table 5 The two-loop integral (95) with $\Lambda_{c}=1 / 2$ multiplied by $-m^{2} \tau / \pi^{4}$ as a function of $\rho:=4 / \tau$. Numbers obtained with $10^{9}$ MC points. Statistical errors between parentheses

\begin{tabular}{ll}
\hline$\rho$ & MC result \\
\hline-1.5 & $3.325(3)-i 1(2) \times 10^{-3}$ \\
-0.5 & $4.817(4)+i 1(3) \times 10^{-3}$ \\
-0.1 & $6.297(6)+i 4(7) \times 10^{-3}$ \\
-0.01 & $7.029(9)-i 1(1) \times 10^{-2}$ \\
-0.001 & $7.16(1)+i 1(3) \times 10^{-2}$ \\
0.001 & $7.234(7)-i 2(3) \times 10^{-2}$ \\
0.01 & $7.358(5)-i 1.3(2) \times 10^{-1}$ \\
0.1 & $7.932(3)-i 9.30(8) \times 10^{-1}$ \\
0.5 & $8.990(3)-i 3.981(4)$ \\
1.5 & $3.60(2) \times 10^{-1}-i 9.464(2)$ \\
\hline
\end{tabular}

gles

$C_{L}:=C\left(\tau, \sigma_{1}+\mu_{1}, \sigma_{0}+\mu_{0}, \mu_{2}, \mu_{3}, \mu_{4}\right)$,

$C_{R}:=C\left(\tau, \sigma_{1}+\mu_{1}, \sigma_{0}+\mu_{0}, \mu_{5}, \mu_{6}, \mu_{7}\right)$,

by means of $\Phi_{1}$,

$m^{4} S_{3}\left(\left\{\mu_{k}\right\}\right)=f \prod_{j=0}^{1}\left(\frac{d \sigma_{j}}{\sigma_{j}+i \epsilon}\right) \Phi_{1}^{\left(\mu_{0}, \mu_{1}\right)}\left[C_{L} C_{R}\right]$.

Now the integrand vanishes fast enough at large $\sigma_{0,1}$, so that no subtraction is needed. Our results for the case $\left\{\mu_{k}\right\}=$ $\{1,1,0,0,0,0,0,0\}$ are shown in Fig. 8, where we compare them with digitized curves obtained from Fig. 4 of [10]. The agreement is good, but our points tend to overshoot the lines at large $\tau$. However, the quality of the plot in Ref. [10] is poor there and the digitization may be misleading. Thus, we cross-checked internally our high-energy results by comparing the outcome of two independent MCs based on method 1 and 2 of Sect. 2, respectively. We did 




Fig. 7 The three-loop self-energy diagram $S_{3}\left(\left\{\mu_{k}\right\}\right)$ with masses $\left\{m_{k}^{2} / m^{2}\right\}=\left\{\mu_{k}\right\}:=\left\{\mu_{0}, \mu_{1}, \mu_{2}, \mu_{3}, \mu_{4}, \mu_{5}, \mu_{6}, \mu_{7}\right\}$

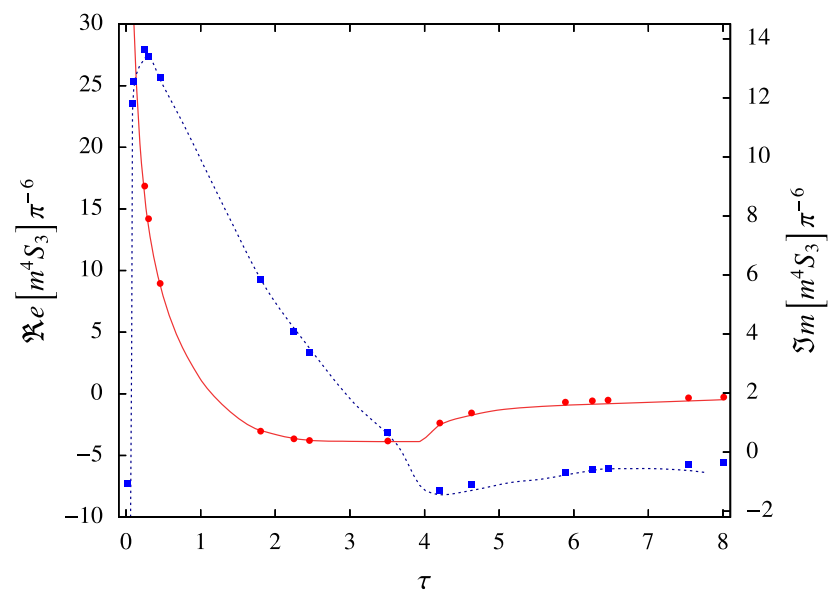

Fig. 8 The three-loop integral $m^{4} S_{3}(1,1,0,0,0,0,0,0)$ as a function of $\tau$. Red bullets (blue squares) refer to the real (imaginary) part computed with $10^{9} \mathrm{MC}$ shots per point. The solid and dashed lines are obtained from [10]. To compare, we divided (not multiplied, as stated in [10]) our Eq. (99) by $\pi^{6}$

not find any systematic difference in the range $7<\tau<50$. Finally, we observe that dealing with arbitrary mass configurations poses no difficulties whatsoever. For example, when $\left\{\mu_{k}\right\}=\{1,1,2,3,4,5,6,7\}$, using OneLOop [16] to evaluate the $C_{L, R}$ triangles gives, at $\tau=10$ and with $10^{9}$ points,

$\frac{m^{4}}{\pi^{6}} S_{3}\left(\left\{\mu_{k}\right\}\right)=1.1453(8) \times 10^{-1}-i 4.11(1) \times 10^{-2}$.

\section{Planar two-loop vertex}

Consider now the two-loop scalar vertex of Fig. 9. Gluing the one-loop triangle on the left

$C_{L}:=C\left(\tau, \sigma_{1}+\mu_{1}, \sigma_{0}+\mu_{0}, \mu_{3}, \mu_{4}, \mu_{5}\right)$

by means of $\Phi_{2}$ gives

$$
m^{4} V_{2}\left(\left\{\mu_{k}\right\}\right)=f \prod_{j=0}^{1}\left(\frac{d \sigma_{j}}{\sigma_{j}+i \epsilon}\right) \Phi_{2}^{\left(\mu_{0}, \mu_{1}, \mu_{2}\right)}\left[C_{L}\right] .
$$

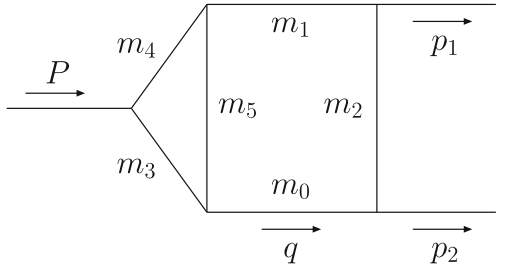

Fig. 9 The planar two-loop vertex $V_{2}\left(\left\{\mu_{k}\right\}\right)$ of (100) with masses $\left\{m_{k}^{2} / m^{2}\right\}=\left\{\mu_{k}\right\}:=\left\{\mu_{0}, \mu_{1}, \mu_{2}, \mu_{3}, \mu_{4}, \mu_{5}\right\}$

This representation holds true with any sign of $\tau, \tau_{1}, \tau_{2}$ and for any choice of internal masses. ${ }^{8}$ In addition, it does not require subtracting large $\sigma_{0,1}$ configurations. In Table 6 we collect a few results obtained by computing the triangle with OneLOop. The last row refers to the Standard-Model-like $Z \rightarrow v \bar{v}$ case with $m_{0}=m_{1}=m_{e}, m_{2}=m_{3}=m_{4}=M_{W}$, $m_{5}=0, P^{2}=M_{Z}^{2}$ and $p_{1,2}^{2}=0$. This shows that the $\mathrm{MC}$ error is under control also for configurations with large mass gaps. Note that $V_{2}\left(\left\{\mu_{k}\right\}\right)$ can also be obtained by gluing together the box on the right $D_{R}:=D\left(\tau_{1}, \tau_{2}, \sigma_{3}+\mu_{3}, \sigma_{4}+\right.$ $\left.\mu_{4}, \tau, \sigma_{b}^{34}, \mu_{1}, \mu_{2}, \mu_{0}, \mu_{5}\right)$ and the tree-level decay on the left. When $k^{2}$ is greater than zero one has

$m^{4} V_{2}\left(\left\{\mu_{k}\right\}\right)=f \prod_{j=3}^{4}\left(\frac{d \sigma_{j}}{\sigma_{j}+i \epsilon}\right) \int_{-1}^{1} \frac{d c_{\theta}}{2} \Phi_{1}^{\left(\mu_{3}, \mu_{4}\right)}\left[D_{R}\right]$.

The loop momentum of the $m_{3}$ line of Fig. 9 flows through just one propagator of $D_{R}$. Because of that, a "technical" cut $\sqrt{\tau^{2} /\left(\tau^{2}+\left(\sigma_{3}-\sigma_{4}\right)^{2}\right)}<\Lambda$ is required to damp the inaccurate large $\sigma_{3,4}$ behaviour of (101). By power counting, the discarded contribution is of $\mathcal{O}\left(\Lambda^{4}\right)$. With $\Lambda=0.05$ and $10^{9}$ MC points (101) reproduces the numbers of Table 6 at the percent level.

\section{Non-planar two-loop vertex}

The same reasoning leading to (101) allows one to compute the non-planar two-loop vertex of Fig. 10,

$$
\begin{aligned}
& m^{4} V_{2}^{n p}\left(\left\{\mu_{k}\right\}\right) \\
& =f \prod_{j=3}^{4}\left(\frac{d \sigma_{j}}{\sigma_{j}+i \epsilon}\right) \int_{-1}^{1} \frac{d c_{\theta}}{2} \Phi_{1}^{\left(\mu_{3}, \mu_{4}\right)}\left[D^{n p}\right],
\end{aligned}
$$

where

$$
\begin{aligned}
D^{n p}: & =D\left(\tau_{1}, \sigma_{3}+\mu_{3}, \tau_{2}, \sigma_{4}\right. \\
& \left.+\mu_{4}, \sigma_{a}^{34}, \sigma_{b}^{34}, \mu_{1}, \mu_{2}, \mu_{0}, \mu_{5}\right) .
\end{aligned}
$$

8 The inclusion of complex masses is in principle possible, although a dedicated study is needed in this case to assess the numerical accuracy, especially for small width-to-mass ratios. 
Table 6 The planar two-loop integral (100) as a function of arbitrary input parameters. Numbers obtained with $10^{9}$ MC points. Statistical errors in parentheses

\begin{tabular}{lllll}
\hline$\tau$ & $\tau_{1}$ & $\tau_{2}$ & $\left\{\mu_{k}\right\}$ & MC result \\
\hline 10 & 2 & 3 & $\{1,2,3,4,5,6\}$ & $-2.751(7)-i 6.729(7)$ \\
-100 & -4 & 7 & $\{0,1,12,7,8,9\}$ & $-1.025(1) \times 10^{-1}+i 1.5(7) \times 10^{-4}$ \\
50 & 30 & 30 & $\{6,23,2,9,9,9\}$ & $9.307(4) \times 10^{-1}-i 1.347(4) \times 10^{-1}$ \\
-40 & 1 & 1 & $\{0,0,0,0,0,0\}$ & $5.918(6)-i 9.51(3)$ \\
1000 & 0 & 0 & $\{1,1,1,1,1,1\}$ & $-3.558(4) \times 10^{-3}+i 2.3557(8) \times 10^{-2}$ \\
$31.9 \times 10^{9}$ & 0 & 0 & $\left\{1,1,24.7 \times 10^{9}, 24.7 \times 10^{9}, 24.7 \times 10^{9}, 0\right\}$ & $-2.5(8) \times 10^{-22}-i 1.7947(1) \times 10^{-19}$ \\
\hline
\end{tabular}



Fig. 10 The non-planar two-loop vertex $V_{2}^{n p}\left(\left\{\mu_{k}\right\}\right)$ of (102) with masses $\left\{m_{k}^{2} / m^{2}\right\}=\left\{\mu_{k}\right\}:=\left\{\mu_{0}, \mu_{1}, \mu_{2}, \mu_{3}, \mu_{4}, \mu_{5}\right\}$

Table 7 The non-planar two-loop integral (102) as a function of $\tau$ with equal internal masses $\left\{\mu_{k}\right\}=\{1,1,1,1,1,1\}$ and massless external momenta $\tau_{1}=\tau_{2}=0$. Numbers obtained with $10^{9} \mathrm{MC}$ points by computing $D^{n p}$ with OneLOop. Statistical errors within parentheses

\begin{tabular}{ll}
\hline$\tau$ & MC result \\
\hline 2.1 & $-1.538(8) \times 10^{1}-i 6(6) \times 10^{-2}$ \\
10 & $8.7(1) \times 10^{-1}-i 2.5415(9) \times 10^{1}$ \\
100 & $1.8848(6)+i 7.469(6) \times 10^{-1}$ \\
1000 & $2.660(2) \times 10^{-2}+i 7.788(2) \times 10^{-2}$ \\
\hline
\end{tabular}

Now the loop momentum of the $m_{3}$ line flows through two propagators of $D^{n p}$. This provides an additional damping factor in (102) with respect to (101), so that large $\sigma_{3,4}$ configurations do not lead to numerical inaccuracies and no technical cut is required. A few numerical results are collected in Table 7.

\section{Planar and non-planar double box}

The planar and non-planar two-loop double boxes are depicted in Fig. 11a, b, respectively. They read

$B_{2}\left(\left\{\mu_{k}\right\}\right)=\frac{1}{m^{6}} f \prod_{j=0}^{1}\left(\frac{d \sigma_{j}}{\sigma_{j}+i \epsilon}\right) \Phi_{3}^{\left(\mu_{0}, \mu_{1}, \mu_{3}\right)}\left[D_{L}\right]$,

$B_{2}^{n p}\left(\left\{\mu_{k}\right\}\right)=\frac{1}{m^{6}} f \prod_{j=0}^{1}\left(\frac{d \sigma_{j}}{\sigma_{j}+i \epsilon}\right) \Phi_{3}^{\left(\mu_{0}, \mu_{1}, \mu_{3}\right)}\left[D_{R}\right]$,
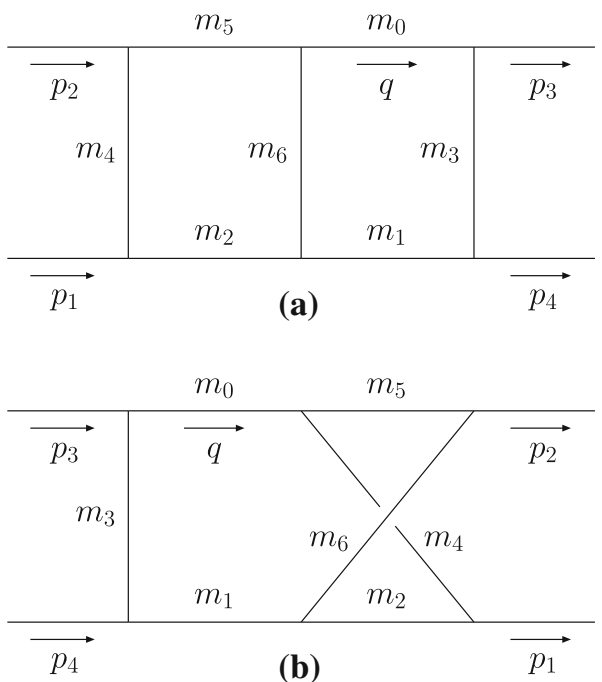

Fig. 11 The planar (a) and non-planar (b) two-loop double boxes $B_{2}\left(\left\{\mu_{k}\right\}\right)$ and $B_{2}^{n p}\left(\left\{\mu_{k}\right\}\right)$ of (103) and (104) with masses $\left\{m_{k}^{2} / m^{2}\right\}=$ $\left\{\mu_{k}\right\}:=\left\{\mu_{0}, \mu_{1}, \mu_{2}, \mu_{3}, \mu_{4}, \mu_{5} \mu_{6}\right\}$

where

$D_{L}:=D\left(\tau_{1}, \tau_{2}, \sigma_{0}+\mu_{0}, \sigma_{1}+\mu_{1}, \tau, \sigma_{b}^{01}, \mu_{2}, \mu_{4}, \mu_{5}, \mu_{6}\right)$,

$D_{R}:=D\left(\tau_{1}, \sigma_{0}+\mu_{0}, \tau_{2}, \sigma_{1}+\mu_{1}, \sigma_{a}^{01}, \sigma_{b}^{01}, \mu_{2}, \mu_{4}, \mu_{5}, \mu_{6}\right)$,

are the one-loop boxes on the left and right sides of Fig. 11a, b, respectively. In Figs. 12 and 13 we present a comparison between our estimates and the results presented in [7] for the case

$\mu_{k}=\{1,1,1,3.24,3.24,1,3.24\}, \tau_{i}=1, \chi=-4$,

$m=50 \mathrm{GeV}$,

in the region of $\tau$ where $\left|\cos \theta_{13}\right| \leq 1 .{ }^{9}$ The agreement is very good. As benchmark values, we list in Table 8 the MC entries of Figs. 12 and 13, together with their statistical errors.

More complex structures

${ }_{9}$ An analytic continuation to nonphysical configurations is possible, although we did not try it. 


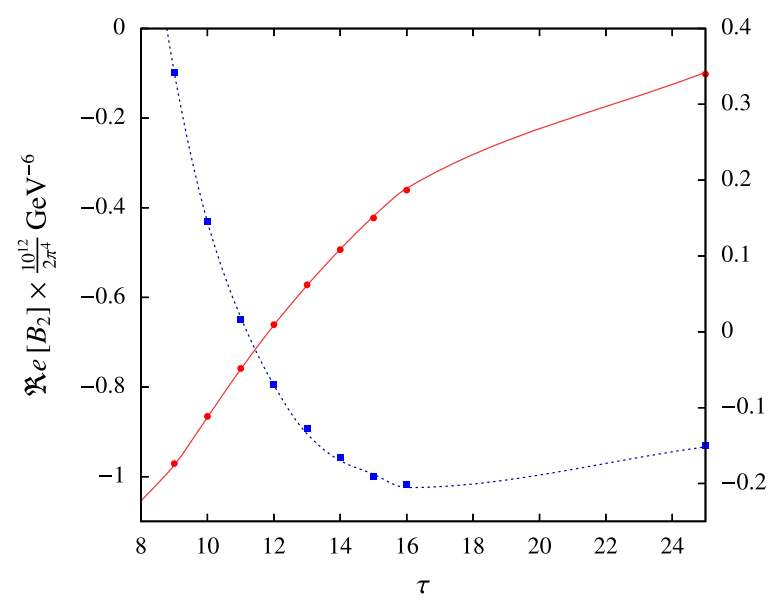

Fig. 12 The two-loop integral $B_{2}\left(\left\{\mu_{k}\right\}\right)$ of (103) as a function of $\tau$ with the input values listed in (105). Red bullets (blue squares) refer to the real (imaginary) part obtained with $10^{9} \mathrm{MC}$ shots per point using OneLOop to compute $D_{L}$. The solid and dashed lines are derived from Fig. 2 of [7]

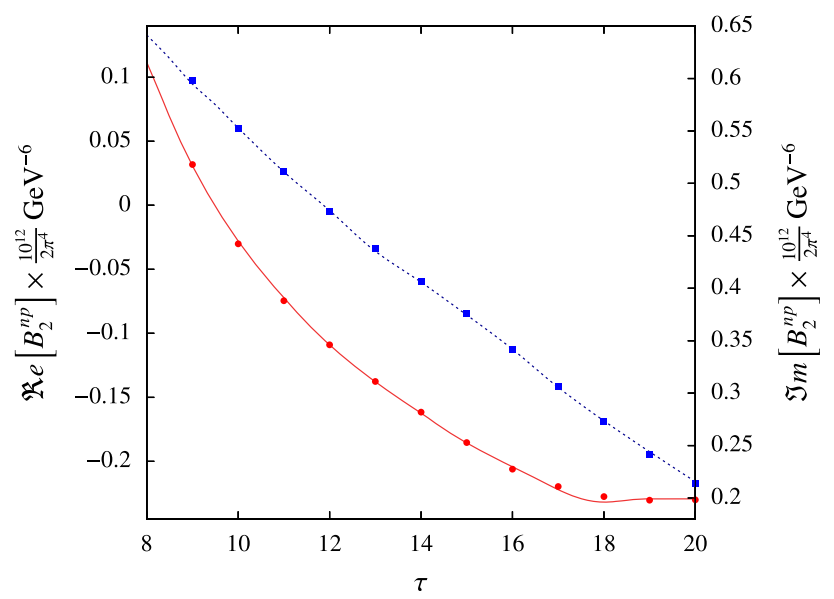

Fig. 13 The two-loop integral $B_{2}^{n p}\left(\left\{\mu_{k}\right\}\right)$ of (104) as a function of $\tau$ with the input values listed in (105) (not $\left\{\mu_{k}\right\}=$ $\{1,1,1,3.24,3.24,3.24,1\}$, as stated in the text of [7]). Red bullets (blue squares) refer to the real (imaginary) part obtained with $10^{9} \mathrm{MC}$ shots per point using OneLOop to compute $D_{R}$. The solid and dashed lines are derived from Fig. 3 of [7]

In all cases presented so far we could perform an easy analytic integration over at least one angular variable of the loop momentum $\omega$. When the number of loops and legs increases, integrating analytically over $c_{\theta}$ and/or $\phi$ is not trivial any more, so that the number of numerical integrations required by the gluing procedure reaches its maximum value, i.e. four. In this paragraph we give a few examples of the gluing approach in these more complex situations. In particular, we use the multichannel approach of Sect. 2.1 to study the scalar triple box $B_{3}$ and two-loop pentabox $E_{2}$ of Figs. 14 and 15, respectively.
Table 8 The MC entries of Figs. 12 and 13 in units of $\mathrm{GeV}^{-6}$. The MC errors are indicated between parentheses

\begin{tabular}{lll}
\hline$\tau$ & $10^{12} B_{2} /\left(2 \pi^{4}\right)$ & $10^{12} B_{2}^{n p} /\left(2 \pi^{4}\right)$ \\
\hline 9 & $-0.971(1)+i 0.342(1)$ & $0.0318(9)+i 0.5985(9)$ \\
10 & $-0.8655(8)+i 0.1457(8)$ & $-0.0301(7)+i 0.5521(7)$ \\
11 & $-0.7586(6)+i 0.0168(6)$ & $-0.0746(6)+i 0.5110(5)$ \\
12 & $-0.6607(7)-i 0.0692(6)$ & $-0.1090(5)+i 0.4729(5)$ \\
13 & $-0.5720(5)-i 0.1275(5)$ & $-0.1376(4)+i 0.4380(4)$ \\
14 & $-0.4934(4)-i 0.1659(4)$ & $-0.1616(3)+i 0.4065(3)$ \\
15 & $-0.4229(3)-i 0.1903(3)$ & $-0.1853(3)+i 0.3763(3)$ \\
16 & $-0.3607(3)-i 0.2019(3)$ & $-0.2061(3)+i 0.3414(2)$ \\
17 & - & $-0.2196(2)+i 0.3065(2)$ \\
18 & - & $-0.2275(2)+i 0.2728(2)$ \\
19 & - & $-0.2303(2)+i 0.2418(2)$ \\
20 & - & $-0.2301(2)+i 0.2138(2)$ \\
25 & $-0.1017(1)-i 0.1506(1)$ & - \\
\hline
\end{tabular}

As for $B_{3}$, inserting (63) into the integrand gives

$$
\begin{aligned}
m^{8} B_{3}= & f \prod_{j=0}^{1}\left(\frac{d \sigma_{j}}{\sigma_{j}+i \epsilon}\right) \int_{-1}^{1} \frac{d c_{\theta}}{2} \int_{0}^{2 \pi} \frac{d \phi}{2 \pi} \\
& \times \Phi_{1}^{\left(\mu_{0}, \mu_{1}\right)}\left[D_{L}\left(c_{\theta}\right) D_{R}\left(c_{\theta}, s_{\phi}\right)\right],
\end{aligned}
$$

where

$$
\begin{aligned}
D_{L}\left(c_{\theta}\right):= & D\left(\sigma_{1}+\mu_{1}, \sigma_{0}+\mu_{0}, \tau_{2}, \tau_{1}, \tau, \sigma_{b}^{01},\right. \\
& \left.\mu_{2}, \mu_{3}, \mu_{4}, \mu_{5}\right), \\
D_{R}\left(c_{\theta}, s_{\phi}\right):= & D\left(\sigma_{1}+\mu_{1}, \sigma_{0}+\mu_{0}, \tau_{3}, \tau_{4}, \tau, \sigma_{c}^{01},\right. \\
& \left.\mu_{6}, \mu_{7}, \mu_{8}, \mu_{9}\right),
\end{aligned}
$$

are the one-loop boxes on the left and right sides of Fig. 14. The change of variable

$y=c_{\phi}$

produces

$$
\begin{aligned}
m^{8} B_{3}= & \frac{1}{8 \tau} f \prod_{j=0}^{1}\left(\frac{d \sigma_{j}}{\sigma_{j}+i \epsilon}\right) \lambda^{\frac{1}{2}} \theta(\lambda) \int_{-1}^{1} d c_{\theta} D_{L}\left(c_{\theta}\right) \\
& \times \int_{-1}^{1} \frac{d y}{\sqrt{1-y^{2}}} \sum_{n=0}^{1} D_{R}\left(c_{\theta},(-1)^{n} \sqrt{1-y^{2}}\right) .
\end{aligned}
$$

When $\tau=50, \chi=-4, \tau_{i}=\mu_{0}=\mu_{1}=1, \mu_{2 \div 9}=0,(109)$ gives, with $10^{9} \mathrm{MC}$ shots and using OneLOop to evaluate $D_{L, R}$,

$m^{8} B_{3}=-9.393(9)-i$ 0.374(9).

Note that it is not difficult to deal with non-planar configurations. For instance, the diagram obtained from Fig. 14 by 


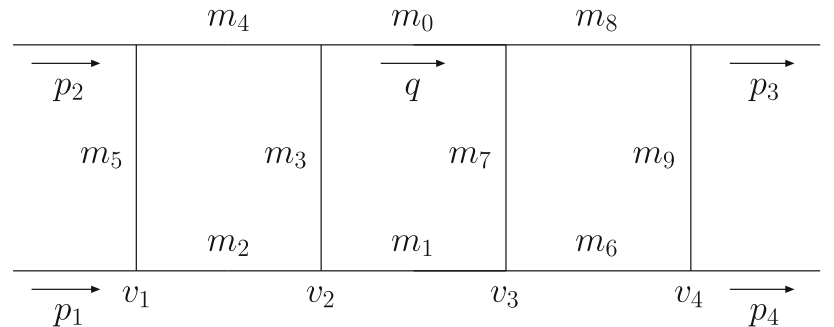

Fig. 14 The three-loop planar box $B_{3}$ of (106)

interchanging the vertices $v_{1} \leftrightarrow v_{2}$ and $v_{3} \leftrightarrow v_{4}$ is as in (106), but with

$$
\begin{aligned}
D_{L}\left(c_{\theta}\right):= & D\left(\tau_{1}, \sigma_{0}+\mu_{0}, \tau_{2}, \sigma_{1}+\mu_{1}, \sigma_{a}^{01}, \sigma_{b}^{01},\right. \\
& \left.\mu_{2}, \mu_{3}, \mu_{4}, \mu_{5}\right), \\
D_{R}\left(c_{\theta}, s_{\phi}\right):= & D\left(\tau_{4}, \sigma_{0}+\mu_{0}, \tau_{3}, \sigma_{1}+\mu_{1}, \sigma_{d}^{01}, \sigma_{c}^{01},\right. \\
& \left.\mu_{6}, \mu_{7}, \mu_{8}, \mu_{9}\right) .
\end{aligned}
$$

Finally, the pentabox depicted in Fig. 15 reads

$$
\begin{aligned}
m^{8} E_{2}= & f \prod_{j=0}^{1}\left(\frac{d \sigma_{j}}{\sigma_{j}+i \epsilon}\right) \int_{-1}^{1} \frac{d c_{\theta}}{2} \int_{0}^{2 \pi} \frac{d \phi}{2 \pi} \\
& \times \Phi_{1}^{\left(\mu_{0}, \mu_{1}\right)}\left[\frac{E(\omega)}{\sigma_{2}+i \epsilon}\right],
\end{aligned}
$$

where $E(\omega)$ is the one-loop pentagon on the right side, which depends upon ten independent invariants - built from the momenta $q=m \omega, p_{3 \div 5}-$ and the five masses $m_{3 \div 7}$. The presence of the $1 /\left(\sigma_{2}+i \epsilon\right)$ propagator forces one to trade the integral over $c_{\theta}$ for an integration over $\sigma_{2}$ to be dealt with the method of Sect. 2. This, together with the change of variable of (108), gives

$$
\begin{aligned}
m^{8} E_{2}= & \frac{1}{4 \tau k} f \prod_{j=0}^{2}\left(\frac{d \sigma_{j}}{\sigma_{j}+i \epsilon}\right) \theta(\lambda) \theta\left(1-\left|c_{\theta}\right|\right) \\
& \times \int_{-1}^{1} \frac{d y}{\sqrt{1-y^{2}}} \sum_{n=0}^{1} E\left(\omega_{n}\right),
\end{aligned}
$$

where

$$
\begin{aligned}
k \lambda^{\frac{1}{2}} c_{\theta}= & \sigma_{0}+\mu_{0}+\sigma_{1}+\mu_{1}-2\left(\sigma_{2}+\mu_{2}-\tau_{2}\right)-\tau \\
& +\sigma_{\tau} \frac{\tau_{12}}{\tau}, \\
\omega_{0,1}:= & \frac{1}{2 \sqrt{\tau}}\left(\sigma_{\tau}, \lambda^{\frac{1}{2}} c_{\theta}, \pm \lambda^{\frac{1}{2}} s_{\theta} \sqrt{1-y^{2}}, \pm \lambda^{\frac{1}{2}} s_{\theta} y\right), \\
\sigma_{\tau}:= & \tau+\sigma_{0}+\mu_{0}-\sigma_{1}-\mu_{1} .
\end{aligned}
$$

To provide a benchmark value, we have chosen to evaluate (113) with $\mu_{0 \div 2}=1, \mu_{3 \div 7}=0$ at a particular $p_{1}+p_{2} \rightarrow$ $p_{3}+p_{4}+p_{5}$ phase-space point satisfying $\tau=50, \tau_{1}=$ $\tau_{2}=0$ and $\tau_{3 \div 5}=1$, namely

$$
\frac{p_{1}}{m}=\frac{\sqrt{\tau}}{2}(1,1,0,0), \quad \frac{p_{2}}{m}=\frac{\sqrt{\tau}}{2}(1,-1,0,0),
$$



Fig. 15 The two-loop planar pentabox $E_{2}$ of (112)

$\frac{p_{3}}{m}=\frac{3}{8} \sqrt{\tau}(1, r, r, 0)$,

$\frac{p_{4}}{m}=\frac{3}{8} \sqrt{\tau}\left[1, r\left(c_{34}-\frac{s_{34}}{\sqrt{2}}\right), r\left(c_{34}+\frac{s_{34}}{\sqrt{2}}\right), r s_{34}\right]$,

with $r=\sqrt{193 / 450}, c_{34}=-159 / 193$ and $s_{34}=\sqrt{1-c_{34}^{2}}$. We employed CutTools [18] to reduce $E\left(\omega_{n}\right)$ to one-loop boxes. Computing the latter with OneLOOp gives, with $10^{9}$ MC shots,

$m^{8} E_{2}=0.1125(1)-i 0.0041(1)$.

Once again, non-planar configurations are obtained without extra effort. For instance, if the vertex $v$ of Fig. 15 is moved to the $m_{3}$ line, (112) still holds by simply modifying the oneloop pentagon $E(\omega)$ accordingly.

\section{UV divergences}

We deal with ultraviolet divergent integrals following the FDR approach of [13], in which the divergent configurations are extracted from the original integrand by partial fractioning. ${ }^{10}$ The resulting expressions are integrable in four dimensions and nicely match the algorithm of Sect. 2 . We illustrate our procedure by means of the scalar two-point function of Fig. 16,

$B_{\mathrm{FDR}}=\int\left[d^{4} q\right] \frac{1}{\bar{D}_{0} \bar{D}_{1}}$,

where

$\bar{D}_{0}:=\bar{q}^{2}-m_{0}^{2}, \quad \bar{D}_{1}:=\bar{q}^{2}-M_{1}^{2}(q)$,

with $M_{1}^{2}(q):=m_{1}^{2}+2(q \cdot P)-P^{2}$ and $\bar{q}^{2}:=q^{2}-\mu^{2}+i \epsilon$. By partial fractioning ${ }^{11}$

$\frac{1}{\bar{D}_{0} \bar{D}_{1}}=\left[\frac{1}{\bar{q}^{4}}\right]+\frac{m_{0}^{2}}{\bar{q}^{2} \bar{D}_{0} \bar{D}_{1}}+\frac{M_{1}^{2}(q)}{\bar{q}^{4} \bar{D}_{1}}$.

\footnotetext{
$\overline{10}$ At one loop, FDR is equivalent to the $\overline{\mathrm{MS}}$ scheme of Dimensional Regularization.

11 The UV divergent piece is dubbed vacuum and written between square brackets by convention.
} 


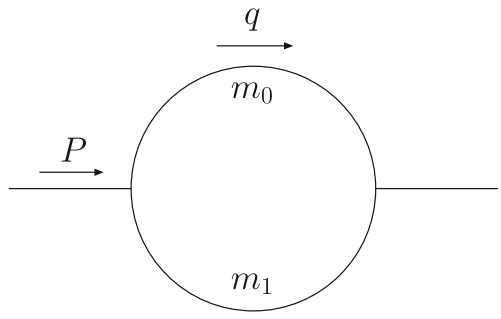

Fig. 16 The one-loop self-energy diagram of (116)

This gives, by definition of FDR integration,

$B_{\mathrm{FDR}}:=\left.\lim _{\mu \rightarrow 0} \int d^{4} q\left(\frac{m_{0}^{2}}{\bar{q}^{2} \bar{D}_{0} \bar{D}_{1}}+\frac{M_{1}^{2}(q)}{\bar{q}^{4} \bar{D}_{1}}\right)\right|_{\mu=\mu_{\mathrm{R}}}$,

where $\mu_{\mathrm{R}}$ is the finite renormalization scale. It is convenient to take the limit $\mu \rightarrow 0$ directly at the integrand level and substitute $\mu$ with $\mu_{\mathrm{R}}$ only in the logarithms. This is achieved by rewriting

$B_{\mathrm{FDR}}=\int d^{4} q\left\{\frac{1}{D_{0} D_{1}}-\frac{1}{\left(q^{2}-\mu_{\mathrm{R}}^{2}+i \epsilon\right)^{2}}\right\}$,

where $D_{i}:=\bar{D}_{i}+\mu^{2}$. By doing so, $\mu^{2}$ is dropped everywhere, except in the logarithmically UV divergent part of the vacuum, where it is replaced by $\mu_{\mathrm{R}}^{2}$. In this way, no $\mu \rightarrow 0$ limit is required, so that (120) is a good starting point for a numerical treatment. In what follows we describe how the methods of Sects. 3 and 4 can be adapted to deal with (120). More complex multi-loop UV divergent configurations can be treated likewise.

\subsection{Integrating over the loop energy component}

We take $m_{1}=m_{0}=m$ for simplicity. A rescaling as in (47) produces

$B_{\mathrm{FDR}}=4 \pi \int_{0}^{\infty} \rho^{2} d \rho I_{t}$,

where

$$
\begin{aligned}
I_{t}:= & \int_{-\infty}^{+\infty} d t\left\{\frac{1}{\left(t^{2}-R_{0}^{2}+i \epsilon\right)\left((t-\sqrt{\tau})^{2}-R_{0}^{2}+i \epsilon\right)}\right. \\
& \left.-\frac{1}{\left(t^{2}-R_{v}^{2}+i \epsilon\right)^{2}}\right\},
\end{aligned}
$$

with

$R_{0}^{2}:=\rho^{2}+1, \quad R_{v}^{2}:=\rho^{2}+v, \quad v:=\mu_{\mathrm{R}}^{2} / m^{2}$.

The Cauchy integral theorem allows one to compute

$$
\begin{aligned}
I_{t}= & \frac{i \pi}{2}\left\{\frac{1}{\left(R_{0}-i \epsilon\right)\left(R_{0}-\sqrt{\tau} / 2-i \epsilon\right)\left(R_{0}+\sqrt{\tau} / 2-i \epsilon\right)}\right. \\
& \left.-\frac{1}{\left(R_{v}-i \epsilon\right)^{3}}\right\} .
\end{aligned}
$$

Inserting this in (121) and using $r=R_{0}-\sqrt{\tau} / 2$ as a new integration variable gives

$B_{\mathrm{FDR}}=2 i \pi^{2} f d r \frac{F(r)}{r-i \epsilon}$,

where

$$
\begin{aligned}
F(r)= & \theta(r-1+\sqrt{\tau} / 2) \sqrt{(r+\sqrt{\tau} / 2)^{2}-1} \\
& \times\left\{\frac{1}{r+\sqrt{\tau}}-r \frac{r+\sqrt{\tau} / 2}{\left[(r+\sqrt{\tau} / 2)^{2}-1+v\right]^{3 / 2}}\right\} .
\end{aligned}
$$

If $\sqrt{\tau}>2$ the pole at $r=i \epsilon$ migrates towards the integration contour when $\epsilon \rightarrow 0$. Treating this with our numerical approach produces the results collected in Table 9.

Integrals with a polynomial degree of divergence can be treated in exactly the same way. As an example, Appendix B details the case of the one-point function

$A_{\mathrm{FDR}}=\int\left[d^{4} q\right] \frac{1}{\bar{D}_{0}}$.

\subsection{Gluing substructures}

The gluing approach of Sect. 4 can be easily extended to (116). Inserting (63) in (120) gives

$B_{\mathrm{FDR}}(\nu)=\frac{\pi}{2 \tau} f \prod_{j=0}^{1}\left(\frac{d \sigma_{j}}{\sigma_{j}+i \epsilon}\right) \lambda^{\frac{1}{2}} \theta(\lambda) J(\nu)$,

with

$J(v)=1-\frac{\sigma_{0} \sigma_{1}}{\left(\sigma_{0}+\mu_{0}-v+i \epsilon\right)^{2}}$

and $v$ defined in (123). The presence of a double pole is an obstacle to a direct numerical treatment of (128). In fact, our algorithm is designed to deal with single poles only. However, we observe that, if $v$ has a finite imaginary part, the singularity never approaches the real axis. In particular, $B_{\mathrm{FDR}}(-i v)$ is better suited than $B_{\mathrm{FDR}}(v)$ to be evaluated numerically if $v \in$ $\Re$. Besides, the connection between the two can be derived by differentiating (120),

$\frac{\partial B_{\mathrm{FDR}}}{\partial \mu_{\mathrm{R}}^{2}}=-2 \int \frac{d^{4} q}{\left(q^{2}-\mu_{\mathrm{R}}^{2}+i \epsilon\right)^{3}}=\frac{i \pi^{2}}{\mu_{\mathrm{R}}^{2}}$,

which gives

$B_{\mathrm{FDR}}(v)=B_{\mathrm{FDR}}\left(-i v^{\prime}\right)-\frac{\pi^{3}}{2}-i \pi^{2} \ln \frac{v^{\prime}}{v}$.

$B_{\mathrm{FDR}}\left(-i v^{\prime}\right)$ still suffers from numerical inaccuracies of type (76) (a). To cure this, we locally subtract from it an approximant, $\tilde{B}_{\mathrm{FDR}}\left(-i v^{\prime}\right)=0$, constructed in such a way that, after changing variables as in (79), all cuts and poles lie in the lower $\sigma_{0}$ complex half-plane. This is obtained by replacing 
Table 9 The integral of (116) with $m=m_{0}=m_{1}=2 \mu_{\mathrm{R}}$ as a function of $\sqrt{\tau}$. The numerical estimates are computed by sampling (125) with $10^{8} \mathrm{MC}$ shots and the analytic results are obtained with OneLOop. MC errors between parentheses

\begin{tabular}{lll}
\hline$\sqrt{\tau}$ & Numerical result & Analytic result \\
\hline 0.2 & $2(2) \times 10^{-6}-i 1.3614(3) \times 10^{1}$ & $0-i 1.3616 \times 10^{1}$ \\
0.4 & $2(2) \times 10^{-6}-i 1.3411(3) \times 10^{1}$ & $0-i 1.3415 \times 10^{1}$ \\
0.6 & $2(2) \times 10^{-6}-i 1.3065(3) \times 10^{1}$ & $0-i 1.3068 \times 10^{1}$ \\
1.5 & $2(2) \times 10^{-6}-i 8.705(1)$ & $0-i 8.7063$ \\
1.9 & $4(4) \times 10^{-7}-i 2.0737(3)$ & $0-i 2.0739$ \\
2.1 & $-9.455(1)+i 4.162(1)$ & $-9.4541+i 4.1616$ \\
4 & $-2.6854(3) \times 10^{1}-i 1.6453(5) \times 10^{1}$ & $-2.6852 \times 10^{1}-i 1.6456 \times 10^{1}$ \\
10 & $-3.0377(5) \times 10^{1}-i 3.828(2) \times 10^{1}$ & $-3.0380 \times 10^{1}-i 3.8280 \times 10^{1}$ \\
50 & $-3.0990(6) \times 10^{1}-i 7.112(5) \times 10^{1}$ & $-3.0981 \times 10^{1}-i 7.1094 \times 10^{1}$ \\
100 & $-3.1009(6) \times 10^{1}-i 8.473(7) \times 10^{1}$ & $-3.1000 \times 10^{1}-i 8.4825 \times 10^{1}$ \\
\hline
\end{tabular}

Table 10 The integral of (131) with $v=1 / 4$ and $\mu_{0}=\mu_{1}=1$. The estimates are obtained by sampling with $10^{8} \mathrm{MC}$ shots (132) evaluated at $v^{\prime}=1$. MC errors between parentheses

\begin{tabular}{ll}
\hline$\sqrt{\tau}$ & $B_{\mathrm{FDR}}\left(-i v^{\prime}\right)-\frac{\pi^{3}}{2}-i \pi^{2} \ln \frac{v^{\prime}}{v}$ \\
\hline 0.2 & $-4(4) \times 10^{-3}-i 1.357(3) \times 10^{1}$ \\
0.4 & $-3(3) \times 10^{-2}-i 1.342(2) \times 10^{1}$ \\
0.6 & $-1(1) \times 10^{-2}-i 1.308(2) \times 10^{1}$ \\
1.5 & $-1(1) \times 10^{-2}-i 8.69(2)$ \\
1.9 & $2(2) \times 10^{-3}-i 2.04(2)$ \\
2.1 & $-9.45(1)+i 4.19(2)$ \\
4 & $-2.688(2) \times 10^{1}-i 1.655(3) \times 10^{1}$ \\
10 & $-3.039(4) \times 10^{1}-i 3.825(5) \times 10^{1}$ \\
50 & $-3.100(8) \times 10^{1}-i 7.12(1) \times 10^{1}$ \\
100 & $-3.09(1) \times 10^{1}-i 8.49(2) \times 10^{1}$ \\
\hline
\end{tabular}

in $(128) \lambda^{\frac{1}{2}} \theta(\lambda) \rightarrow \sqrt{\lambda^{2}-4 \tau i \epsilon} \theta\left(\lambda+4 \tau \sigma_{0}\right)$. In summary, we rewrite

$$
\begin{aligned}
B_{\mathrm{FDR}}\left(-i \nu^{\prime}\right)= & \frac{\pi}{2 \tau} f \prod_{j=0}^{1}\left(\frac{d \sigma_{j}}{\sigma_{j}+i \epsilon}\right) \\
& \times\left[\lambda^{\frac{1}{2}} \theta(\lambda)-\sqrt{\lambda^{2}-4 \tau i \epsilon} \theta\left(\lambda+4 \tau \sigma_{0}\right)\right] J\left(-i \nu^{\prime}\right) .
\end{aligned}
$$

In Table 10 we present our estimates for $B_{\mathrm{FDR}}(1 / 4)$ with $\mu_{0}=\mu_{1}=1$ obtained by means of Eqs. (131) and (132). The figures match the results of Table 9, although with larger errors. However, we point out that the gluing method is more flexible when it comes to generic kinematics. For instance, with $\tau=-10, \mu_{0}=1, \mu_{1}=4$, one obtains, with $10^{8} \mathrm{MC}$ points,

$B_{\mathrm{FDR}}(1 / 4)=0.02(2)-i 27.20(3)$,

to be compared to the analytic value $-i 27.220$.

\section{IR divergences}

We deal with IR divergent integrals by means of the FDR approach of [19], where a small mass $\mu$, added to judiciously chosen propagators, is used as a regulator of both infrared and collinear divergences. In this section, we illustrate how this allows one to combine virtual and real contributions prior to integration. After that, our method can be used to evaluate numerically loop integrals where the IR configurations are locally subtracted. We study, in particular, the IR divergent scalar triangle

$$
\begin{aligned}
C_{\mathrm{IR}} & :=\lim _{\mu \rightarrow 0} \int d^{4} q \frac{1}{D_{0} D_{1} D_{2}}, \\
D_{0} & :=q^{2}-\mu^{2}+i \epsilon, \\
D_{1} & :=\left(q+p_{1}\right)^{2}-\mu^{2}+i \epsilon, D_{2}:=\left(q-p_{2}\right)^{2}-\mu^{2}+i \epsilon,
\end{aligned}
$$

that appears in a $P \rightarrow p_{1}+p_{2}$ decay with $p_{i}^{2}=0$ and $s:=P^{2}$. However, our findings can be generalized to more complex environments.

Our strategy is based on combining together cut-diagrams that are individually divergent, but whose sum is finite. We use a scalar massless $g \varphi^{3}$ theory defined through the Feynman rules of Fig. 17,

where we have introduced propagators with positive and negative values of the energy and the momentum component along the $x$ direction. The cuts contributing to $\varphi^{*} \rightarrow \varphi \varphi(\varphi)$ are listed in Fig. 18 where, to make contact with (134),

$\mathrm{D}_{\mathrm{a}}+\mathrm{D}_{\mathrm{e}}=4 \pi^{2} g^{4} \frac{\pi}{2}\left(i C_{\mathrm{IR}}\right)$,

$\mathrm{D}_{\mathrm{c}}+\mathrm{D}_{\mathrm{g}}=4 \pi^{2} g^{4} \frac{\pi}{2}\left(i C_{\mathrm{IR}}\right)^{*}$.

The diagrams are organized in pairs sharing collinear singularities. For instance, in $\mathrm{D}_{\mathrm{a}}$ the energy component of propagator 1 is the sum of those of propagators 2 and 3. Thus, propagators 1 and 3 never pinch in the $q_{1}^{0}$ complex plane, and propagator 3 can only become collinear to 4 . Likewise, in $\mathrm{D}_{\mathrm{b}}$ 


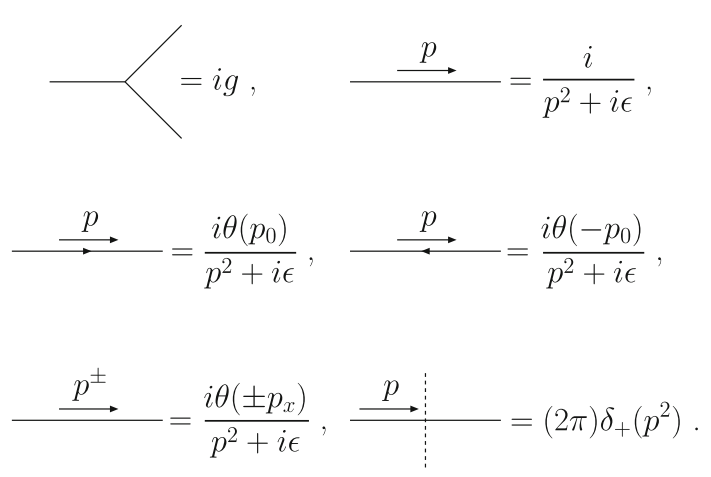

Fig. 17 The Feynman rules of the $g \varphi^{3}$ theory. A special notation is used for propagators with positive and negative values of $p_{0}$ and $p_{x}$, which, in our convention, coincides with the direction of the back-toback final-state particles in the $P$ rest-frame. The complex conjugate of such rules is used in the r.h.s. of diagrams cut by a dashed line
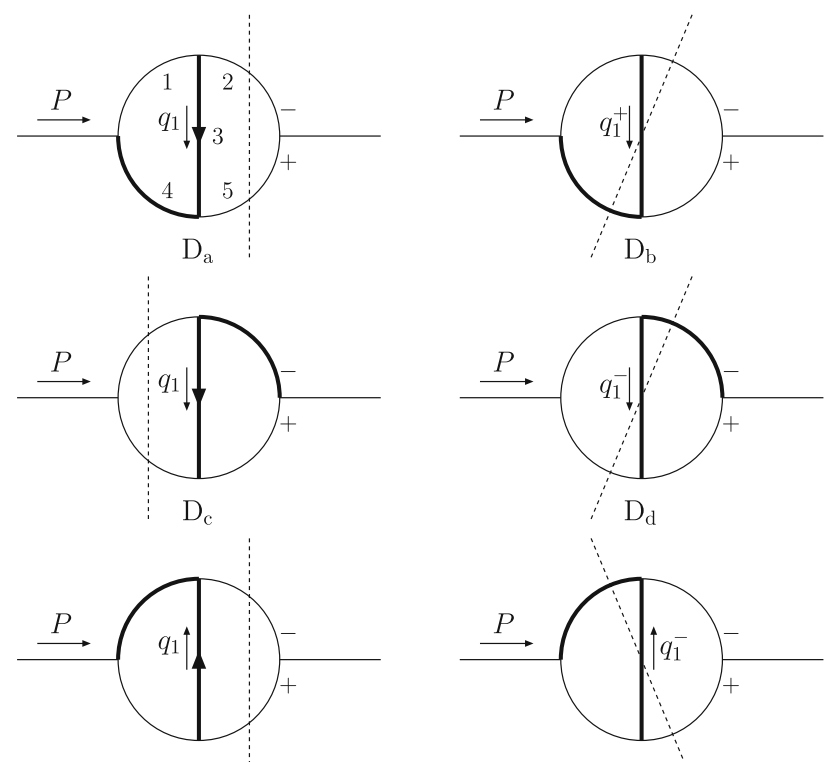

$\mathrm{D}_{\mathrm{e}}$
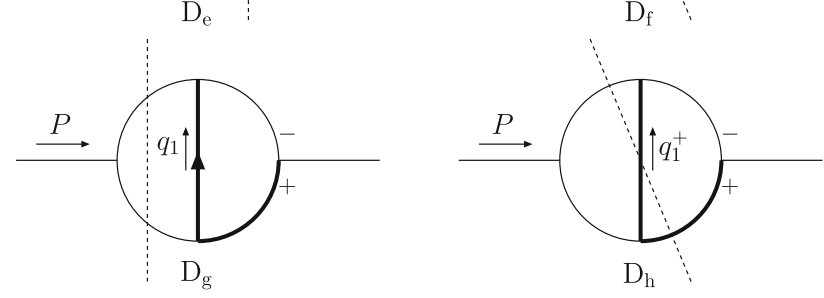

Fig. 18 The two- and three-particle cuts contributing to $\varphi^{*} \rightarrow \varphi \varphi(\varphi)$ in the $P$ rest frame where the propagators 2 and 5 have negative and positive components of the momentum along $x$, as indicated by the $\mp$ labels attached to them. Thick lines represent the propagators to which $\mu^{2} \rightarrow 0$ is added, as explained in the text

the sign of the momentum components along $x$ only allows particles 3 and 4 to become collinear to 5 . In both cases, we regulate the singular splitting by including a small mass $\mu$ in propagators 3 and 4 , leaving 5 massless. ${ }^{12}$ In summary,

\footnotetext{
$\overline{12}$ Note that adding $\mu^{2}$ also to 1 and/or 2 does not change the asymptotic $\mu \rightarrow 0$ limit of the result. This is used, for instance, in (135).
}

$D_{a}+D_{b}$ is free of collinear divergences, and the same happens for $D_{c}+D_{d}$. In addition, $D_{a}+D_{b}+D_{c}+D_{d}$ is also free of infrared singularities. A similar reasoning applies to $D_{e}+D_{f}+D_{g}+D_{h}$, but with an opposite sign of the energy component of $q_{1}$. The previous analysis shows that the threeparticle cuts $\mathrm{D}_{\mathrm{b}}$ and $\mathrm{D}_{\mathrm{d}}$ can be used as local countertems for $\mathrm{D}_{\mathrm{a}}+\mathrm{D}_{\mathrm{c}} \cdot{ }^{13}$ This requires common reference frames. One can employ two different routings for $\mathrm{D}_{\mathrm{a}}+\mathrm{D}_{\mathrm{b}}$ and $\mathrm{D}_{\mathrm{c}}+\mathrm{D}_{\mathrm{c}}$. However, they must coincide in the limit $q_{1} \rightarrow 0$ to guarantee the cancellation of the soft behaviour of $D_{a}+D_{b}+D_{c}+D_{d}$. In particular, when computing $\mathrm{D}_{\mathrm{a}, \mathrm{b}}$ we assign a momentum $q_{2}$ to propagator 4 , from left to right, and choose

$\omega_{1}:=q_{1} / \sqrt{s}=\left(t_{1}, \rho_{1}, 0,0\right)$,
$\omega_{2}:=q_{2} / \sqrt{s}=\left(t_{2}, \rho_{2} c_{\theta}, \rho_{2} s_{\theta} s_{\phi}, s_{\theta} c_{\phi}\right)$.

On the other hand, we calculate $\mathrm{D}_{\mathrm{c}, \mathrm{d}}$ with $q_{2}$ assigned to propagator 5 and

$\omega_{1}=\left(t_{1},-\rho_{1}, 0,0\right)$,
$\omega_{2}=\left(t_{2}, \rho_{2} c_{\theta}, \rho_{2} s_{\theta} s_{\phi}, s_{\theta} c_{\phi}\right)$.

The result of the computation is reported in Appendix $\mathrm{C}$ in terms of integrals over

$R_{i}:=\sqrt{\rho_{i}^{2}+\eta}$, with $\eta:=\mu^{2} / s$.

It is convenient to further split $\mathrm{D}_{\mathrm{a}, \mathrm{c}}=\mathrm{D}_{\mathrm{a}, \mathrm{c}}^{\mathrm{s}}+\mathrm{D}_{\mathrm{a}, \mathrm{c}}^{\mathrm{u}}$, where the superscripts ${ }^{\mathrm{s}, \mathrm{u}}$ refer to the subtracted and unsubtracted regions, which correspond to the integration intervals $\sqrt{\eta}<$ $R_{1}<1 / 2$ and $1 / 2<R_{1}<\infty$, respectively. In fact, $\mathrm{D}_{\mathrm{b}, \mathrm{d}}$ contribute in the subtracted region only, and $\mathrm{D}_{\mathrm{a}}^{\mathrm{u}}+\mathrm{D}_{\mathrm{c}}^{\mathrm{u}}$ is free of IR singularities,

$$
\begin{aligned}
\frac{s}{g^{4}}\left(\mathrm{D}_{\mathrm{a}}^{\mathrm{u}}+\mathrm{D}_{\mathrm{c}}^{\mathrm{u}}\right) & =-2 \pi^{5}\left(\ln ^{2} 2+2 \operatorname{Li}_{2}(-1 / 2)\right) \\
& =254.838137 \cdots .
\end{aligned}
$$

An analytic calculation [19] shows that $\sum_{\mathrm{j}=\mathrm{a}, \mathrm{b}, \mathrm{c}, \mathrm{d}} D_{j}=0$. Hence, one must have

$$
K:=\frac{s}{g^{4}}\left(\mathrm{D}_{\mathrm{a}}^{\mathrm{s}}+\mathrm{D}_{\mathrm{c}}^{\mathrm{s}}+\mathrm{D}_{\mathrm{b}}+\mathrm{D}_{\mathrm{d}}\right)=-254.838137 \cdots .
$$

In Table 11 we display our numerical estimate of $K$ based on Eqs. (C.22), (C.29) and (C.34). The correct result is precisely approached and the MC error does not grow when decreasing $\eta$, which is an indication that the local cancellation works as expected. Finally, we point out that the outlined strategy can be turned into a fully exclusive local subtraction algorithm by introducing suitable phase-space mappings, as described in [20].

\footnotetext{
${ }^{13}$ An analogous procedure holds for the last four cuts of Fig. 18.
} 
Table 11 The combination of cut-diagrams defined in (140) as a function of $\eta$ in (138). Numbers obtained with $10^{10}$ MC shots

\begin{tabular}{ll}
\hline$\eta$ & $K$ \\
\hline $10^{-10}$ & $-254.81(1)$ \\
$10^{-11}$ & $-254.83(1)$ \\
$10^{-12}$ & $-254.84(1)$ \\
\hline
\end{tabular}

Table 12 Time to generate $10^{6} \mathrm{MC}$ shots on a single $2.2 \mathrm{GHz}$ processor

\begin{tabular}{lll}
\hline Type of integral & Location & Time [s] \\
\hline One-loop triangle & Last row of Table 2 & 0.25 \\
Two-loop self-energy & Eq. (96) & 4.7 \\
Two-loop vertex & Last row of Table 6 & 16 \\
Planar double box & Last row of Table 8 & 71 \\
Three-loop planar box & Eq.(110) & 22 \\
Two-loop pentabox & Eq.(115) & 17 \\
UV one-loop bubble & Last row of Table 9 & 0.17 \\
IR case & Table 11 & 0.13 \\
\hline
\end{tabular}

\section{Conclusion and outlook}

We have presented a flexible method for the numerical treatment of loop integrals in four-dimensional Minkowski space, without the need of explicit contour deformation. This is achieved by exploiting the $i \epsilon$ prescription with a small finite value of $\epsilon$ and making changes of variables to reduce the variance of both the real and imaginary parts of the integrand. We propose a semi-numerical approach, in which an analytic integration over loop time-components is followed by multichannel Monte Carlo integration. In some cases, further integrations can be performed before the final numerical step. The method lends itself readily to the evaluation of complex multi-loop structures by gluing together simpler substructures. It also deals easily with processes involving many different external and propagator mass scales, where analytical results are difficult to obtain.

In practice, we find that $10^{9}$ Monte Carlo shots with $\epsilon \sim 10^{-7}$ (in terms of some relevant mass scale) can yield relative precision of the order of $10^{-4}$ for one-loop diagrams and $10^{-3}$ for two- and three-loops obtained by gluing together analytical results for one-loop substructures. As for the performance of our algorithms, we report in Table 12 the time to produce $10^{6} \mathrm{MC}$ shots with method 1 for a few representative cases. It ranges from a few tenths of a second to more than a minute. Method 2 gives somewhat slower timings.

We have focused on scalar integrals without any structure in the numerator, but we expect that the treatment of loop tensors should follow the same guidelines described in this paper. In particular, the approach of Sect. 3, in which an analytic integration is performed over the loop time-component, should work as it stands. As for the gluing method of Sect. 4, adding structures in the numerator could potentially lead to worse behaviour that needs to be corrected by local subtractions of large loop configurations, as done in Eqs. (86), (95), (132), or by the technical cuts described in (97) and (101). We leave a detailed study of this subject for further investigation.

We have sketched out how our method can be extended to UV and IR divergent configurations. Again, a deeper investigation is left for the future.

In summary, we believe that a numerical treatment of virtual corrections in four dimensions, of the type we have proposed, could be very beneficial in the computation of complicated multi-leg multi-scale amplitudes. More specifically, we think that the direction to go would be to integrate directly the amplitude as a whole, rather than the separate MIs. This could mitigate some of the large gauge cancellations among individual contributions, if common loop momentum routings are chosen for classes of diagrams. In addition, Monte Carlo integration of the loops and over the phase-space of real emissions can be merged, potentially stabilising and speeding up the calculation.

Acknowledgements The work of RP is supported by the SRA grant PID2019-106087GB-C21 (10.13039/501100011033), by the Junta de Andalucía grants A-FQM-467-UGR18 and P18-FR-4314 (FEDER), and by the COST Action CA16201 PARTICLEFACE. The work of BW was partially supported by STFC HEP consolidated grants ST/P000681/1 and ST/T000694/1.

Data Availability Statement This manuscript has no associated data or the data will not be deposited. [Authors' comment: This is a theoretical paper with no external associated data.]

Open Access This article is licensed under a Creative Commons Attribution 4.0 International License, which permits use, sharing, adaptation, distribution and reproduction in any medium or format, as long as you give appropriate credit to the original author(s) and the source, provide a link to the Creative Commons licence, and indicate if changes were made. The images or other third party material in this article are included in the article's Creative Commons licence, unless indicated otherwise in a credit line to the material. If material is not included in the article's Creative Commons licence and your intended use is not permitted by statutory regulation or exceeds the permitted use, you will need to obtain permission directly from the copyright holder. To view a copy of this licence, visit http://creativecomm ons.org/licenses/by/4.0/.

Funded by $\mathrm{SCOAP}^{3}$.

\section{Appendix A: Taylor expansions}

The Taylor expansions for integrals like (71) and (72) can be obtained using the general result

$$
\begin{aligned}
\int d^{4} q & \frac{q^{\mu_{1}} \ldots q^{\mu_{k}} q^{\nu_{1}} \ldots q^{\nu_{k}}}{\left(q^{2}-m^{2}+i \epsilon\right)^{n}} \\
& =C_{n, k} \frac{i \pi^{2}}{m^{2 n-2 k-4}} g^{\left\{\mu_{1} \nu_{1}\right.} \ldots g^{\left.\mu_{k} \nu_{k}\right\}}
\end{aligned}
$$


where

$C_{n, k}=(-1)^{n}(-4)^{k} \frac{(n-k-3) !}{k !(n-1) !}$,

which can be established by induction.

For (71), we first make a shift of variable $q \rightarrow q+p_{2}$. Then for $\tau_{1}=\tau_{2}=0, \mu_{1}=\mu_{2}=1$ we have

$C=\sum_{k, l=0}^{\infty} f d^{4} q \frac{\left(2 q \cdot p_{1}\right)^{k}\left(-2 q \cdot p_{2}\right)^{l}}{\left(q^{2}-m^{2}+i \epsilon\right)^{k+l+3}}$.

Applying (A.1), and noting that terms with $l \neq k$ vanish as they would involve $\tau_{1}$ or $\tau_{2}$,

$$
\begin{aligned}
\frac{C}{i \pi^{2}}= & \sum_{k=0}^{\infty}(-4)^{k} \frac{C_{2 k+3, k}}{m^{2 k+2}} p_{1}^{\mu_{1}} \ldots p_{1}^{\mu_{k}} p_{2}^{\nu_{1}} \ldots p_{2}^{\nu_{k}} \\
& \times g_{\left\{\mu_{1} \nu_{1}\right.} \ldots g_{\left.\mu_{k} \nu_{k}\right\}} \\
= & -\sum_{k=0}^{\infty} \frac{p_{1}^{\mu_{1}} \ldots p_{1}^{\mu_{k}} p_{2}^{\nu_{1}} \ldots p_{2}^{\nu_{k}}}{(2 k+2) ! m^{2 k+2}} g_{\left\{\mu_{1} \nu_{1}\right.} \ldots g_{\left.\mu_{k} \nu_{k}\right\}} \\
= & \frac{-1}{m^{2}} \sum_{k=0}^{\infty} \frac{(k !)^{2}}{(2 k+2) !} \tau^{k},
\end{aligned}
$$

where $\tau=2 p_{1} \cdot p_{2} / m^{2}$. For $\tau_{1}, \tau_{2} \neq 0$, we have instead of (A.3)

$$
\begin{aligned}
C= & \sum_{k, l=0}^{\infty} f d^{4} q \frac{\left(2 q \cdot p_{1}-p_{1}^{2}\right)^{k}\left(-2 q \cdot p_{2}-p_{2}^{2}\right)^{l}}{\left(q^{2}-m^{2}+i \epsilon\right)^{k+l+3}} \\
= & -\frac{i \pi^{2}}{2 m^{2}}\left[1+\frac{1}{12}\left(\tau+\tau_{1}+\tau_{2}\right)+\frac{1}{90}\left(\tau^{2}+\tau_{1}^{2}+\tau_{2}^{2}\right.\right. \\
& \left.\left.+\tau \tau_{1}+\tau \tau_{2}+\tau_{1} \tau_{2}\right)+\ldots\right] .
\end{aligned}
$$

This expansion can be extended to general masses by the substitutions

$$
\begin{aligned}
& \tau_{1} \rightarrow \tau_{1}+\mu_{2}-\mu_{1}, \\
& \tau_{2} \rightarrow \tau_{2}+\mu_{2}-1 .
\end{aligned}
$$

For the expansion of (72) we have

$$
\begin{aligned}
\frac{D_{0}}{i \pi^{2}}= & \sum_{j, k, l, n} \frac{l !\left(-m^{2} \tau\right)^{l-n}}{n !(l-n) !} \\
& \times f d^{4} q \frac{\left(-2 p_{1} \cdot q\right)^{j}\left(2 p_{2} \cdot q\right)^{k}\left[2\left(p_{3}-p_{1}\right) \cdot q\right]^{n}}{\left(q^{2}-m^{2}+i \epsilon\right)^{j+k+l+4}} .
\end{aligned}
$$

Note that the number of factors of $q^{\mu}$ must be even, $j+k+$ $n=2 K$. Then

$$
\begin{aligned}
\frac{D_{0}}{i \pi^{2}}= & \sum_{j, k, l, n}(-1)^{j+l+n} 4^{K} \frac{l ! \tau^{l-n}}{n !(l-n) !} \frac{C_{N, K}}{m^{2 K+4}} \\
& \times p_{1}^{\mu_{1}} \ldots p_{1}^{\mu_{j}} p_{2}^{\mu_{j+1}} \ldots p_{2}^{\mu_{j+k}} \\
& \times\left(p_{3}-p_{1}\right)^{\mu_{j+k+1}} \ldots\left(p_{3}-p_{1}\right)^{\mu_{2 K}} \\
& \times g_{\left\{\mu_{1} \mu_{2}\right.} \ldots g_{\left.\mu_{2 K-1} \mu_{2 K}\right\}}
\end{aligned}
$$

where $N=j+k+l+4$. Substituting (A.2), we obtain (87).

\section{Appendix B: The one-point FDR integral}

We compute the one-loop integral

$A_{\mathrm{FDR}}=\int\left[d^{4} q\right] \frac{1}{\bar{D}_{0}}$,

with $\bar{D}_{0}$ given in Eq. (117). Extracting the vacuum produces the expansion

$\frac{1}{\bar{D}_{0}}=\left[\frac{1}{\bar{q}^{2}}\right]+\left[\frac{m_{0}^{2}}{\bar{q}^{4}}\right]+\frac{m_{0}^{4}}{\bar{q}^{4} \bar{D}_{0}}$,

hence

$A_{\mathrm{FDR}}=\left.\lim _{\mu \rightarrow 0} \int d^{4} q \frac{m_{0}^{4}}{\bar{q}^{4} \bar{D}_{0}}\right|_{\mu=\mu_{\mathrm{R}}}$.

By taking the $\mu \rightarrow 0$ limit at the integrand level and replacing $\mu$ with $\mu_{\mathrm{R}}$ in the logarithmic divergent vacuum one obtains

$A_{\mathrm{FDR}}=$
$\int d^{4} q\left\{\frac{1}{q^{2}-m_{0}^{2}+i \epsilon}-\frac{1}{q^{2}+i \epsilon}-\frac{m_{0}^{2}}{\left(q^{2}-\mu_{\mathrm{R}}^{2}+i \epsilon\right)^{2}}\right\}$.

Choosing now $m=m_{0}$ gives

$A_{\mathrm{FDR}} / m^{2}=4 \pi \int_{0}^{\infty} \rho^{2} d \rho I_{t}$,

where

$$
\begin{aligned}
I_{t}:= & \int_{-\infty}^{+\infty} d t\left\{\frac{1}{t^{2}-R_{0}^{2}+i \epsilon}-\frac{1}{t^{2}-\rho^{2}+i \epsilon}\right. \\
& \left.-\frac{1}{\left(t^{2}-R_{v}^{2}+i \epsilon\right)^{2}}\right\},
\end{aligned}
$$

with $R_{0}$ and $R_{v}$ defined in (123). One computes

$I_{t}=i \pi\left(\frac{1}{\rho}-\frac{1}{R_{0}}-\frac{1}{2 R_{v}^{3}}\right)$,

which gives

$A_{\mathrm{FDR}} / m^{2}=4 i \pi^{2} f \frac{d \rho}{\rho} \theta(\rho) F(\rho)$,

where

$F(\rho):=\frac{1}{\sqrt{1+\frac{1}{\rho^{2}}}\left(1+\sqrt{1+\frac{1}{\rho^{2}}}\right)}-\frac{1}{2\left(1+\frac{v}{\rho^{2}}\right)^{\frac{3}{2}}}$.

Note that there is no pole in this case. In Table 13 we report a comparison between a numerical implementation of (B.16) and the analytic result

$A_{\mathrm{FDR}} / m^{2}=i \pi^{2}(1+\ln v)$. 
Table 13 The integral of (B.16) as a function of $v$ compared to the analytic result of (B.17). The numerical estimates are obtained with $10^{8}$ shots. MC errors between parentheses

\begin{tabular}{lll}
\hline$v$ & Numerical result & Analytic result \\
\hline 0.1 & $-i 1.2855(2) \times 10^{1}$ & $-i 1.2856 \times 10^{1}$ \\
0.5 & $i 3.0281(4)$ & $i 3.0285$ \\
1 & $i 9.869(1)$ & $i 9.8696$ \\
2 & $i 1.6709(2) \times 10^{1}$ & $i 1.6711 \times 10^{1}$ \\
10 & $i 3.2596(4) \times 10^{1}$ & $i 3.2595 \times 10^{1}$ \\
\hline
\end{tabular}

\section{Appendix C: The IR integrals}

Here we obtain onefold integral representations for the cutdiagrams $D_{a, b, c, d}$ of Sect. 6 and prove (139).

The diagram $\mathrm{D}_{\mathrm{b}}$ :

Choosing the momenta as in (136) gives

$$
\begin{aligned}
\frac{\mathrm{D}_{\mathrm{b}}}{g^{4}}= & \frac{8 \pi^{3}}{s} \int d^{4} \omega_{1} d^{4} \omega_{2} \frac{\delta_{+}\left(\sigma_{2}\right) \delta_{+}\left(\sigma_{3}-\eta\right) \delta_{+}\left(\sigma_{4}-\eta\right)}{\left(\sigma_{1}-\eta+i \epsilon\right)\left(\sigma_{5}-i \epsilon\right)} \\
& \times \theta\left(\rho_{1}+\rho_{2} c_{\theta}\right),
\end{aligned}
$$

with

$$
\begin{aligned}
& \sigma_{1}=\left(1-t_{2}\right)^{2}-\rho_{2}^{2}, \\
& \sigma_{2}=\left(1-t_{1}-t_{2}\right)^{2}-\rho_{1}^{2}-\rho_{2}^{2}-2 \rho_{1} \rho_{2} c_{\theta}, \\
& \sigma_{3}=t_{1}^{2}-\rho_{1}^{2}, \sigma_{4}=t_{2}^{2}-\rho_{2}^{2}, \\
& \sigma_{5}=\sigma_{2}-1+2\left(t_{1}+t_{2}\right) .
\end{aligned}
$$

Note that a harmless $\mu^{2}$ has been added to propagator 1 and that the Heaviside function forces propagator 5 to have a positive component of the momentum along $x$. Using the three Dirac delta functions one arrives at

$$
\begin{aligned}
\frac{\mathrm{D}_{\mathrm{b}}}{g^{4}}= & -\frac{8 \pi^{5}}{s} \int_{\sqrt{\eta}}^{\infty} d R_{1} \int_{\sqrt{\eta}}^{\infty} d R_{2} \frac{1}{1-2 R_{2}} \frac{1}{1-2 R^{+}} \\
& \times \theta\left(1-R^{+}\right) \theta\left(1-\left|c_{\theta}\right|\right) \theta\left(\rho_{1}+\rho_{2} c_{\theta}\right),
\end{aligned}
$$

with $R_{1,2}$ in (138), $R^{+}:=R_{1}+R_{2}$ and

$c_{\theta}=\frac{1}{2 \rho_{1} \rho_{2}}\left[1+2\left(\eta+R_{1} R_{2}-R^{+}\right)\right]$.

Integrating analytically over $R_{2}$ produces logarithms with boundaries determined by the three Heaviside functions. The result reads

$$
\frac{\mathrm{D}_{b}}{g^{4}}=-\frac{2 \pi^{5}}{s} \int_{\sqrt{\eta}}^{\frac{1}{2}} \frac{d R_{1}}{R_{1}} \ln \left(\eta \frac{1+\frac{1-2 R_{1}}{R_{1}+\sqrt{R_{1}^{2}-\eta}}}{R_{1}+\sqrt{R_{1}^{2}-\eta}-\eta}\right) .
$$

The diagram $\mathrm{D}_{a}$ :

We split $\mathrm{D}_{a}$ into two components, $\mathrm{D}_{a}=D_{a}^{+}+D_{a}^{-}$, with positive and negative values of $q_{1}$ along $x$. Choosing the momenta as in (136) produces

$$
\begin{aligned}
\frac{\mathrm{D}_{a}^{+}}{g^{4}}= & \frac{4 i \pi^{2}}{s} \int d^{4} \omega_{1} d^{4} \omega_{2} \prod_{j=1,3,4}\left(\frac{1}{\sigma_{j}-\eta+i \epsilon}\right) \\
& \times \delta_{+}\left(\sigma_{2}\right) \delta_{+}\left(\sigma_{5}\right) \theta\left(\rho_{1}+\rho_{2} c_{\theta}\right),
\end{aligned}
$$

with the same $\sigma_{1 \div 5}$ of (C.19). Using the two delta functions gives

$$
\begin{aligned}
\frac{\mathrm{D}_{a}^{+}}{g^{4}}= & \frac{8 i \pi^{4}}{s} \int_{0}^{\infty} d \rho_{1} \rho_{1} \int_{0}^{\infty} d \rho_{2} \rho_{2} I_{1} \\
& \times \theta\left(1-\left|c_{\theta}\right|\right) \theta\left(\rho_{1}^{2}-\rho_{2}^{2}+1 / 4\right),
\end{aligned}
$$

where

$c_{\theta}=\frac{1}{2 \rho_{1} \rho_{2}}\left(\frac{1}{4}-\rho_{1}^{2}-\rho_{2}^{2}\right)$

and

$$
\begin{aligned}
I_{1}:= & \int_{0}^{\infty} d t_{1}\left\{\frac{1}{t_{1}^{2}-R_{1}^{2}+i \epsilon}\right. \\
& \left.\times \frac{1}{\left(1 / 2+t_{1}\right)^{2}-R_{2}^{2}+i \epsilon} \frac{1}{\left(1 / 2-t_{1}\right)^{2}-R_{2}^{2}+i \epsilon}\right\} .
\end{aligned}
$$

$\mathrm{D}_{a}^{-}$is obtained from (C.24) by replacing

$$
\theta\left(\rho_{1}^{2}-\rho_{2}^{2}+1 / 4\right) \rightarrow \theta\left(-\rho_{1}^{2}+\rho_{2}^{2}-1 / 4\right) .
$$

Hence

$\frac{\mathrm{D}_{a}}{g^{4}}=\frac{8 i \pi^{4}}{s} \int_{0}^{\infty} d \rho_{1} \rho_{1} \int_{0}^{\infty} d \rho_{2} \rho_{2} I_{1} \theta\left(1-\left|c_{\theta}\right|\right)$.

Computing $I_{1}$ with the Cauchy integral theorem gives

$$
\begin{aligned}
& \frac{\mathrm{D}_{a}}{g^{4}}=-\frac{8 \pi^{5}}{s} \int_{\sqrt{\eta}}^{\infty} d R_{1} \int_{\sqrt{\eta}}^{\infty} d R_{2} \theta\left(1-\left|c_{\theta}\right|\right) \\
& \times\left\{\frac{1}{1+2 R_{2}} \frac{1}{1+2 R^{+}}-\frac{1}{1-2 R_{2}+i \epsilon} \frac{1}{1-2 R^{+}+i \epsilon}\right\} .
\end{aligned}
$$

Note the appearance of the same denominator structures of (C.20). An integration over $R_{2}$ produces

$$
\begin{aligned}
\frac{\mathrm{D}_{a}}{g^{4}}= & -\frac{2 \pi^{5}}{s} \int_{\sqrt{\eta}}^{\infty} \frac{d R_{1}}{R_{1}}\left\{\ln \frac{1+2 R_{2}}{1+2 R^{+}}\right. \\
& \left.-\ln \frac{1-2 R_{2}+i \epsilon}{1-2 R^{+}+i \epsilon}\right\}_{R_{2}^{-}}^{R_{2}^{+}},
\end{aligned}
$$

where

$$
R_{2}^{ \pm}:=\sqrt{\left(\frac{1}{2} \pm \sqrt{R_{1}^{2}-\eta}\right)^{2}+\eta} .
$$

The diagram $\mathrm{D}_{c}$ :

It is the complex conjugate of (C.29). 


\section{The diagram $\mathrm{D}_{d}$ :}

Inserting a harmless $\mu^{2}$ in propagator 5 and choosing the momenta as in (137) gives

$$
\frac{\mathrm{D}_{d}}{g^{4}}=\frac{8 \pi^{3}}{s} \int d^{4} \omega_{1} d^{4} \omega_{2} \frac{\delta_{+}\left(\sigma_{2}-\eta\right) \delta_{+}\left(\sigma_{3}-\eta\right) \delta_{+}\left(\sigma_{4}\right)}{\left(\sigma_{1}+i \epsilon\right)\left(\sigma_{5}-\eta-i \epsilon\right)}
$$$$
\times \theta\left(c_{\theta}\right),
$$

where

$\sigma_{1}=\sigma_{4}+1-2\left(t_{2}-t_{1}\right), \quad \sigma_{2}=\left(1-t_{2}\right)^{2}-\rho_{2}^{2}$,

$\sigma_{3}=t_{1}^{2}-\rho_{1}^{2}, \quad \sigma_{4}=\left(t_{2}-t_{1}\right)^{2}-\rho_{1}^{2}-\rho_{2}^{2}-2 \rho_{1} \rho_{2} c_{\theta}$,

$\sigma_{5}=t_{2}^{2}-\rho_{2}^{2}$.

Using the delta functions produces

$$
\begin{aligned}
\frac{\mathrm{D}_{d}}{g^{4}}= & -\frac{8 \pi^{5}}{s} \int_{\sqrt{\eta}}^{\infty} d R_{1} \int_{\sqrt{\eta}}^{\infty} d R_{2} \frac{1}{1-2 R_{2}} \frac{1}{1-2 R^{+}} \\
& \times \theta\left(1-R^{+}\right) \theta\left(c_{\theta}\right) \theta\left(1-c_{\theta}\right),
\end{aligned}
$$

with $c_{\theta}$ as in (C.21). Integrating over $R_{2}$ gives

$\frac{\mathrm{D}_{d}}{g^{4}}=-\frac{2 \pi^{5}}{s} \int_{\sqrt{\eta}}^{R_{1}^{+}} \frac{d R_{1}}{R_{1}} L\left(R_{1}\right)$,

where

$$
\begin{aligned}
L\left(R_{1}\right):= & \ln \left[\left(\frac{1}{1-2 R_{1}}+\frac{1}{R_{1}+\sqrt{R_{1}^{2}-\eta}}\right)\right. \\
& \left.\times \frac{\eta\left(R_{1}-2 \eta\right)}{\left(R_{1}+\sqrt{R_{1}^{2}-\eta}-\eta\right)\left(R_{1}\left(1-2 R_{1}\right)+2 \eta\right)}\right],
\end{aligned}
$$

and

$R_{1}^{+}:=\frac{1}{2} \frac{1+4 \eta^{2}}{1+\sqrt{\eta-4 \eta^{2}(1-\eta)}}$.

The combination $\mathrm{D}_{a}^{u}+D_{c}^{u}$ :

This is obtained from (C.29) and its complex conjugate by replacing the integration region $\sqrt{\eta}<R_{1}<\infty$ by $1 / 2<$ $R_{1}<\infty$. Upon doing so, $\eta$ can be set to 0 and $R_{2}^{ \pm}=R_{1} \pm$ $1 / 2$. Introducing $x=2 R_{1}$ gives
$\frac{\mathrm{D}_{a}^{u}+D_{c}^{u}}{g^{4}}=-4 \frac{\pi^{5}}{s} \int_{1}^{\infty} \frac{d x}{x}\left(\ln \frac{x+2}{x+1}+\ln \left|\frac{x-2}{x-1}\right|\right)$,

from which (139) follows.

\section{References}

1. G. Heinrich, Phys. Rept. 922, 1 (2021). https://doi.org/10.1016/j. physrep.2021.03.006

2. D.E. Soper, Phys. Rev. D 62, 014009 (2000). https://doi.org/10. 1103/PhysRevD.62.014009

3. T. Binoth, G. Heinrich, Nucl. Phys. B 585, 741 (2000). https://doi. org/10.1016/S0550-3213(00)00429-6

4. T. Binoth, J.P. Guillet, G. Heinrich, E. Pilon, C. Schubert, JHEP 10, 015 (2005). https://doi.org/10.1088/1126-6708/2005/10/015

5. Z. Nagy, D.E. Soper, Phys. Rev. D 74, 093006 (2006). https://doi. org/10.1103/PhysRevD.74.093006

6. E. de Doncker, Y. Shimizu, J. Fujimoto, F. Yuasa, Comput. Phys. Commun. 159, 145 (2004). https://doi.org/10.1016/j.cpc.2004.01. 004

7. F. Yuasa, E. de Doncker, N. Hamaguchi, T. Ishikawa, K. Kato, Y. Kurihara, J. Fujimoto, Y. Shimizu, Comput. Phys. Commun. 183, 2136 (2012). https://doi.org/10.1016/j.cpc.2012.05.018

8. E. de Doncker, F. Yuasa, K. Kato, T. Ishikawa, J. Kapenga, O. Olagbemi, Comput. Phys. Commun. 224, 164 (2018). https://doi. org/10.1016/j.cpc.2017.11.001

9. J. Baglio, F. Campanario, S. Glaus, M. Mühlleitner, J. Ronca, M. Spira, J. Streicher, JHEP 04, 181 (2020). https://doi.org/10.1007/ JHEP04(2020)181

10. A. Ghinculov, Phys. Lett. B 385, 279 (1996). https://doi.org/10. 1016/0370-2693(96)00871-4

11. Guillet, J. Ph. and Pilon, E. and Shimizu, Y. and Zidi, M. S., PTEP 2020(4), 043B01 (2020). https://doi.org/10.1093/ptep/ptaa020

12. Bauberger, Stefan and Freitas, Ayres and Wiegand, Daniel, JHEP 01, 024 (2020). https://doi.org/10.1007/JHEP01(2020)024

13. R. Pittau, JHEP 11, 151 (2012). https://doi.org/10.1007/ JHEP11(2012)151

14. R. Kleiss, R. Pittau, Comput. Phys. Commun. 83, 141 (1994). https://doi.org/10.1016/0010-4655(94)90043-4

15. D. Kermanschah, e-Print: arXiv:2110.06869 [hep-ph] (2021)

16. A. van Hameren, Comput. Phys. Commun. 182, 2427 (2011). https://doi.org/10.1016/j.cpc.2011.06.011

17. D.J. Broadhurst, Z. Phys. C 47, 115 (1990). https://doi.org/10. 1007/BF01551921

18. G. Ossola, C.G. Papadopoulos, R. Pittau, JHEP 03, 042 (2008). https://doi.org/10.1088/1126-6708/2008/03/042

19. R. Pittau, Eur. Phys. J. C 74(1), 2686 (2014). https://doi.org/10. 1140/epjc/s10052-013-2686-1

20. C. Gnendiger et al., Eur. Phys. J. C 77(7), 471 (2017). https://doi. org/10.1140/epjc/s10052-017-5023-2 